\title{
A FICTITIOUS DOMAIN METHOD WITH MIXED FINITE ELEMENTS FOR ELASTODYNAMICS*
}

\author{
E. BÉCACHE ${ }^{\dagger}$, J. RODRÍGUEZ ${ }^{\ddagger}$ AND C. TSOGKA ${ }^{\S}$
}

\begin{abstract}
We consider in this paper the wave scattering problem by an object with Neumann boundary conditions in an anisotropic elastic body. To obtain an efficient numerical method (permitting the use of regular grids) we follow a fictitious domain approach coupled with a first order velocity stress formulation for elastodynamics. We first observe that the method does not always converge when the $Q_{1}^{\text {div }}-Q_{0}$ finite element is used. In particular, the method converges for some scattering object geometries but not for others. Note that the convergence of the $Q_{1}^{\text {div }}-Q_{0}$ finite element method was shown in [E. Bécache, P. Joly, and C. Tsogka, SIAM J. Numer. Anal., 39 (2002), pp. 2109-2132] for the elastodynamic problem in the absence of a scattering object (i.e., without the coupling of the mixed finite elements with the fictitious domain method). Therefore we propose here a modification of the $Q_{1}^{\text {div }}-Q_{0}$ element following the approach in [E. Bécache, J. Rodríguez, and C. Tsogka, On the convergence of the fictitious domain method for wave equation problems, Technical report 5802, INRIA, 2006], where the simpler acoustic case was considered. To study the numerical properties of the new element we carry out a dispersion analysis. Several numerical simulations as well as a numerical convergence analysis show that the proposed method provides a good approximate solution.
\end{abstract}

Key words. mixed finite elements, fictitious domain method, elastic waves, convergence

AMS subject classifications. 65M60, 65M12, 65M15, 65C20, 74S05

DOI. $10.1137 / 060655821$

1. Introduction. We consider here the scattering of elastic waves by objects or cracks with a homogeneous Neumann boundary condition. This is of interest in several applications such as ultrasonic nondestructive testing, seismic wave propagation, etc. To develop an efficient numerical method we intend to use a fictitious domain approach. Following this approach, we extend artificially the solution to a fictitious domain with a simple shape, typically a rectangle in $2 \mathrm{D}$. Then the boundary condition on the complex geometry is enforced by introducing an auxiliary unknown that leaves only at the boundary of the object. The key point of the method is that the mesh for the unknowns leaving on the enlarged domain can be chosen independently of the geometry of the object. In particular, one can use regular grids or structured meshes which allows for simple and efficient computations.

The fictitious domain method was initially introduced for stationary problems $[3,30,24,18,20,27]$, and it was then successfully generalized to time-dependent problems. In particular, it was used for modeling unsteady incompressible viscous flow with fixed or moving boundaries [25, 21, 22, 23] and in several applications of wave propagation, such as acoustic scattering problems [15, 10, 12], electromagnetic scattering problems [17], and musical acoustics [33, 29, 4]. For both stationary and time-dependent problems the fictitious domain improves the performance of the nu-

\footnotetext{
* Received by the editors March 30, 2006; accepted for publication (in revised form) January 16 2007; published electronically May 29, 2007.

http://www.siam.org/journals/sisc/29-3/65582.html

†POEMS, INRIA-Rocquencourt, BP 105, F-78153 Le Chesnay Cédex, France (eliane.becache@ inria.fr).

‡POEMS, ENSTA, 32 boulevard Victor, 75739 Paris cedex 15, France (jeronimo.rodriguez@ ensta.fr)

$\S$ Department of Mathematics, University of Chicago, 5734 University Avenue Chicago, IL 60637 (tsogka@math.uchicago.edu).
} 
merical method in terms of computational time and memory. However, the accuracy of the method is limited by construction due to the lack of regularity of the solution in the extended domain [18].

A main feature of the fictitious domain method is that it can be applied only to essential boundary conditions (i.e., that can be considered as an equality constraint in the functional space). In the case we are interested in, the boundary condition is of the Neumann type for the displacement. To apply the fictitious domain method we have to choose a formulation for which the stress tensor $\sigma$ is included in the unknowns and is sought in a subspace of $(H(\operatorname{div}))^{2}$ : the first order velocity-stress system or the second order stress formulation. In this way the boundary condition becomes of the essential type for $\boldsymbol{\sigma}$. We chose the first order formulation because it can be combined with the perfectly matched absorbing layer model, which is an efficient way of simulating wave propagation in infinite domains $[11,16]$.

For efficiency reasons we would like to discretize the velocity-stress system with mixed finite elements that are compatible with mass lumping. This will permit us to obtain an explicit in time discretization scheme. To our knowledge this is not so obvious; the main difficulty appearing in this problem is finding a way to take into account the symmetry of the stress tensor. The approach which is usually followed is the relaxed symmetry approach: The symmetry is imposed in a weak sense via the introduction of a Lagrange multiplier $[1,2,34,35,31]$. Another approach using spaces of symmetric stress tensors, based on composite elements, was introduced in [28]. None of these, however, are adapted to mass lumping. An answer to this difficulty was proposed in [9], where the $Q_{k+1}^{\text {div }}-Q_{k}$ finite element has been introduced.

A nonstandard convergence analysis of the $Q_{k+1}^{\text {div }}-Q_{k}$ elements has been carried out in [7] for their scalar version and in [9] for their elastodynamic vectorial version. However, the convergence analysis presented there deals only with the mixed problem without the scattering object (i.e., without the coupling of the mixed finite elements with the fictitious domain method). Recently, in [10] the authors considered the scalar case and showed through some numerical experiments that this finite element approximation does not always converge when it is coupled with the fictitious domain method. Motivated by this negative result, a new finite element based on the enrichment of the approximation space for the primal unknown (which is the pressure in this case) was introduced. Moreover, in [10] the convergence of this finite element coupled with the fictitious domain method was theoretically proven.

In the present paper we treat the elastodynamic case where the convergence issues are similar to those in the scalar case. Namely, we show through some numerical experiments that the $Q_{1}^{\text {div }}-Q_{0}$ element does not always converge when it is coupled with the fictitious domain method (the method converges for some scattering object geometries, while it does not converge for others). Following the same ideas presented in [10] we introduce a modified finite element for the vectorial case, the so-called $Q_{1}^{\text {div }}-P_{1}^{\text {disc }}$ where we enrich the approximation space for the velocity from $Q_{0}$ to $P_{1}$ discontinuous functions. This amounts to adding four additional degrees of freedom per element for the velocity and results in introducing spurious modes to the solution. To get rid of this nonphysical part of the solution we propose to attenuate the spurious modes by introducing a damping term in the equations. We study (numerically) the error between the discrete and continuous solutions in terms of the damping parameter $(\beta)$ and propose a way to determine $\beta$ in order to minimize this error.

The theoretical convergence of the method is not a straightforward generalization of the one presented in [10] for the scalar case. The main difficulty is due to the lack of regularity of the solution in the extended fictitious domain. However, several 
numerical simulations indicate that the method converges, and we conjecture that this is true. The numerical order of convergence obtained for a particular object is in agreement with the conjectured theoretical one.

The outline of the paper is as follows. In section 2 we present the elastodynamic equations and the fictitious domain formulation that we will use in what follows. In section 3.2 we illustrate with numerical experiments that the $Q_{1}^{\text {div }}-Q_{0}$ element does not always converge. The modified $Q_{1}^{\text {div }}-P_{1}^{\text {disc }}$ element is introduced in section 4 and its dispersion analysis in section 4.3. The proposed method for attenuating the nonphysical part of the solution is then presented in section 4.4 , and the difficulties of the theoretical convergence analysis are explained in section 4.2. Numerical results that indicate the convergence of the method are shown in section 4.5. Finally in section 5 we consider a numerical convergence analysis for a particular object.

\section{The fictitious domain formulation of the diffraction problem.}

2.1. The continuous problem. We consider the diffraction of an elastic wave by a two-dimensional object with a free surface boundary condition (Neumann boundary condition on the velocity field $\mathbf{v}$ ) on its boundary $\Gamma$. The object can be either an obstacle with a closed boundary or a crack with an open boundary (see Figure 2.1), but for the sake of clarity we will consider here only this second configuration. The domain of propagation is denoted by $\Omega$ with an exterior boundary $\Sigma$ (see Figure 2.1), and we assume that $C=\Omega \cup \Gamma$ is a domain of "simple" geometry, typically a rectangle. Under the assumptions of small deformations, wave propagation can be modeled by the velocity-stress formulation of the linear elastodynamic equations (e.g., [9] or [13] for the static case)

$$
\left\{\begin{array}{ccccc}
\rho \frac{\partial \mathbf{v}}{\partial t}-\operatorname{div} \boldsymbol{\sigma} & =\mathbf{f} & \text { in } \Omega, & \text { (a) } \\
\mathbf{A} \frac{\partial \boldsymbol{\sigma}}{\partial t}-\varepsilon(\mathbf{v}) & =0 & \text { in } \Omega, & \text { (b) } \\
\boldsymbol{\sigma} \mathbf{n} & & 0 & \text { on } \Gamma, & \text { (c) } \\
\mathbf{v} & = & 0 & \text { on } \Sigma, & \text { (d) }
\end{array}\right.
$$

together with the initial conditions

$$
\mathbf{v}(t=0)=\mathbf{v}_{0}, \quad \boldsymbol{\sigma}(t=0)=\boldsymbol{\sigma}_{0} .
$$

In (2.1), the unknowns are the vector $\mathbf{v} \in \mathbb{R}^{2}$ and the second order symmetric tensor $\boldsymbol{\sigma} \in \mathbb{R}^{2 \times 2}$ (i.e., $\boldsymbol{\sigma}=\boldsymbol{\sigma}^{t}$ ). These two quantities represent, respectively, the velocity

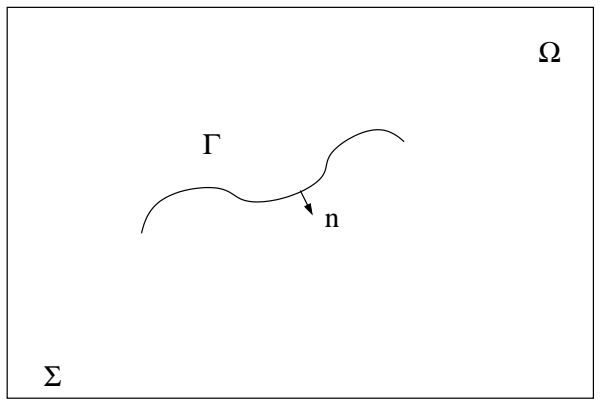

FIG. 2.1. Geometry of the problem. 
field and the stress tensor. The scalar function $\rho$ and the tensor $\mathbf{A}$ characterize the medium, and the vector field $\mathbf{f}$ represents the external forces. Moreover, we assume that $\rho$ satisfies

$$
0<\rho^{-} \leq \rho(x) \leq \rho^{+}<+\infty,
$$

and $\mathbf{A}$ is a fourth order symmetric positive tensor such that

$$
0<\kappa|\boldsymbol{\tau}|^{2} \leq \mathbf{A}(x) \boldsymbol{\tau}: \boldsymbol{\tau} \leq \nu|\boldsymbol{\tau}|^{2} \quad \forall \boldsymbol{\tau} \text { symmetric, } \quad \boldsymbol{\tau} \neq 0 .
$$

This formulation is preferred to the classical displacement formulation, because the boundary condition on the crack is natural (of Neumann type) for the displacement while it becomes essential on $\boldsymbol{\sigma}$, and the fictitious domain approach can then be followed.

We also assume that the support of the initial data $\left(\mathbf{v}_{0}, \boldsymbol{\sigma}_{0}\right)$ and the support of the source $\mathbf{f}$ do not intersect $\Gamma$, which means that

$$
\operatorname{supp}\left(\mathbf{v}_{0}\right) \cup \operatorname{supp}\left(\boldsymbol{\sigma}_{0}\right) \subset C \backslash \Gamma, \quad \bigcup_{t \leq T} \operatorname{supp}(\mathbf{f}(t)) \subset C \backslash \Gamma .
$$

The classical variational formulation of (2.1) would be set in some functional spaces that depend on the shape of the obstacle (i.e., depend on $\Omega$ ). More precisely, the classical formulation is

$$
\left\{\begin{array}{lll}
\text { Find }(\mathbf{v}(t), \boldsymbol{\sigma}(t)) \in \underline{\underline{X}}_{0}^{\text {sym }} \times \underline{M} \text { satisfying } & & \\
\frac{\mathrm{d}}{\mathrm{d} t} \int_{\Omega} \mathbf{A} \boldsymbol{\sigma}: \boldsymbol{\tau} \mathrm{d} x+\int_{\Omega} \operatorname{div} \boldsymbol{\tau} \cdot \mathbf{v} \mathrm{d} x=0 & \forall \boldsymbol{\tau} \in \underline{\underline{X}}_{0}^{s y m}, \\
\frac{\mathrm{d}}{\mathrm{d} t} \int_{\Omega} \rho \mathbf{v} \cdot \mathbf{w d} x-\int_{\Omega} \operatorname{div} \boldsymbol{\sigma} \cdot \mathbf{w} \mathrm{d} x & =\int_{\Omega} \mathbf{f} \cdot \mathbf{w} \mathrm{d} x & \forall \mathbf{w} \in \underline{M}, \\
(\boldsymbol{\sigma}, \mathbf{v})_{t=0} & =\left(\boldsymbol{\sigma}_{0}, \mathbf{v}_{0}\right), &
\end{array}\right.
$$

where the functional spaces are defined as

$$
\begin{aligned}
& \underline{X}=H(\operatorname{div} ; \Omega), \quad \underline{\underline{X}}=\underline{X} \times \underline{X}, \quad \underline{\underline{X}}^{\text {sym }}=\{\boldsymbol{\tau} \in \underline{\underline{X}}: \boldsymbol{\tau} \text { is symmetric }\} \\
& \underline{\underline{X}}_{0}^{\text {sym }}=\left\{\boldsymbol{\tau} \in \underline{\underline{X}}^{\text {sym }}: \boldsymbol{\tau} \mathbf{n}=0 \text { on } \Gamma\right\}, \quad M=L^{2}(\Omega), \quad \underline{M}=M \times M .
\end{aligned}
$$

The well-posedness of the problem (2.4) results from the classical theory on hyperbolic PDEs.

Theorem 2.1. Let $\mathbf{f} \in C^{0}([0, T], \underline{M}), \sigma_{0} \in \underline{\underline{X}}_{0}^{\text {sym }}(\Omega), \mathbf{v}_{0} \in \underline{M}$ satisfying (2.3). Then problem (2.4) has a unique solution $(\boldsymbol{\sigma}, \mathbf{v}) \in\left(C^{1}\left([0, T],\left(L^{2}(\Omega)\right)^{2 \times 2}\right) \cap\right.$ $\left.C^{0}\left([0, T], \underline{\underline{X}}_{0}^{\text {sym }}(\Omega)\right)\right) \times C^{1}([0, T], \underline{M})$.

The fictitious domain formulation of this problem consists in taking into account the boundary condition on $\Gamma$ in a weak way, by introducing a Lagrange multiplier $\boldsymbol{\lambda}$ defined on $\Gamma$. This allows for working in functional spaces (for the volume unknowns) which do not depend any more on the shape of the obstacle. The fictitious domain formulation is then the following (to simplify the notations, we will denote by 
$(\boldsymbol{\sigma}(t), \mathbf{v}(t))$ the new unknowns defined now in $C)$ :

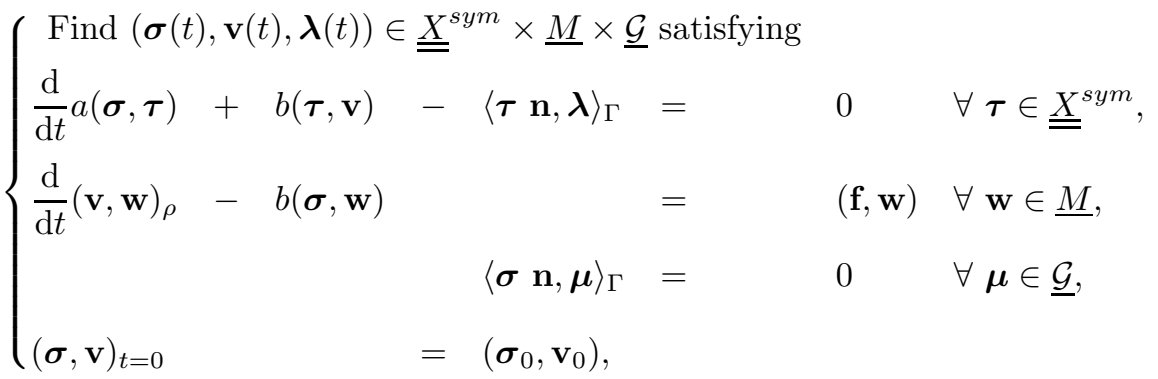

where the functional spaces are now defined as

$$
\begin{aligned}
& \underline{X}=H(\operatorname{div} ; C), \quad \underline{\underline{X}}=\underline{X} \times \underline{X}, \quad \underline{\underline{X}}{ }^{\text {sym }}=\{\boldsymbol{\tau} \in \underline{\underline{X}}: \tau \text { is symmetric }\}, \\
& M=L^{2}(C), \quad \underline{M}=M \times M, \quad \mathcal{G}=H_{00}^{1 / 2}(\Gamma), \quad \underline{\mathcal{G}}=\mathcal{G} \times \mathcal{G},
\end{aligned}
$$

the bilinear forms as

$$
\left\{\begin{array}{rlrl}
a(\boldsymbol{\sigma}, \boldsymbol{\tau})=\int_{C} \mathbf{A} \boldsymbol{\sigma}: \boldsymbol{\tau} \mathrm{d} x & & \forall(\boldsymbol{\sigma}, \boldsymbol{\tau}) \in \underline{\underline{X}} \times \underline{\underline{X}}, \\
(\mathbf{v}, \mathbf{w})_{\eta}=\int_{C} \eta \mathbf{v} \cdot \mathbf{w d} x & \forall(\mathbf{v}, \mathbf{w}) \in \underline{M} \times \underline{M}, \\
b(\boldsymbol{\tau}, \mathbf{w})=\int_{C} \operatorname{div}(\boldsymbol{\tau}) \cdot \mathbf{w d} x & \forall \underline{\underline{X}} \times \underline{M},
\end{array}\right.
$$

and the bracket $\langle\boldsymbol{\sigma} \mathbf{n}, \boldsymbol{\mu}\rangle_{\Gamma}$ is the duality product between $\underline{\mathcal{G}}$ and $\underline{\mathcal{G}}^{\prime}$. Note that, due to assumption (2.3), the data also belong to

$$
\mathbf{f} \in C^{0}([0, T], \underline{M}), \quad \boldsymbol{\sigma}_{0} \in \underline{\underline{X}}(C), \quad \mathbf{v}_{0} \in \underline{M} .
$$

In the following we will denote by $(\cdot, \cdot):=(\cdot, \cdot)_{1}$ the usual $L^{2}(C)$ scalar product.

Remark 1 (on the regularity of the solution). For regular data, one can expect more regularity on the solution. However, in general, the space regularity of the solution is at most

$$
\boldsymbol{\sigma}(t) \in\left(H^{\frac{1}{2}-\varepsilon}(\operatorname{div}, C)\right)^{2}, \quad \mathbf{v}(t) \in\left(H^{\frac{1}{2}-\varepsilon}(C)\right)^{2}, \quad \varepsilon>0,
$$

and this is obtained for regular enough data and a regular geometry of the crack. This is due to the fact that the unknowns are defined on the whole domain $C$ without considering the geometry of the obstacle, so that $\mathbf{v}$ has now a jump in $C$.

The regularity in $\Omega$ (i.e., outside the obstacle) is, in general, higher and depends on the geometry of the obstacle. In particular, for data $\left(\boldsymbol{\sigma}_{0}, \mathbf{v}_{0}, \mathbf{f}\right)$ satisfying $(2.3)$, we have

- for a closed boundary

$$
\mathbf{v} / \Omega(t) \in\left(H^{2}(\Omega)\right)^{2}, \quad \boldsymbol{\lambda}(t) \in\left(H^{3 / 2}(\Gamma)\right)^{2} ;
$$

- for an open boundary, due to the singular behavior near the tip of the crack [26] (the solution behaves as $\sqrt{r}, r$ being here the distance to the tip), we have

$$
\mathbf{v}_{\mid \Omega}(t) \in\left(H^{3 / 2-\varepsilon}(\Omega)\right)^{2}, \quad \boldsymbol{\lambda}(t) \in\left(H^{1-\varepsilon}(\Gamma)\right)^{2}, \quad \varepsilon>0 .
$$


2.2. The semidiscrete approximation. For the approximation in space of this problem, we introduce finite dimensional spaces $\underline{\underline{X}}^{\text {sym }} \subset \underline{\underline{X}}^{\text {sym }}, \underline{M}_{h} \subset \underline{M}$, and $\underline{\mathcal{G}}_{H} \subset \underline{\mathcal{G}}$ satisfying the approximation properties

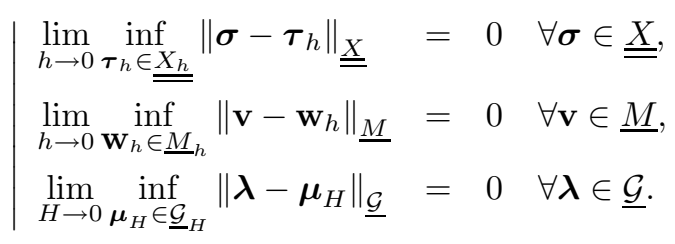

The semidiscrete problem is then

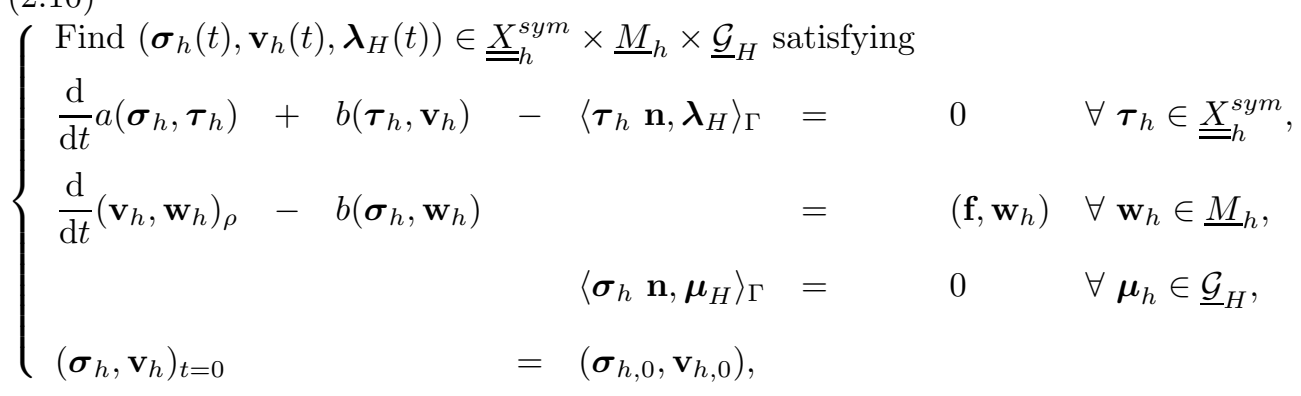

where $\left(\boldsymbol{\sigma}_{h, 0}, \mathbf{v}_{h, 0}\right) \in \underline{\underline{X}}_{h} \times \underline{M}_{h}$ is an approximation of the exact initial condition.

Now the question is how we can choose the approximate spaces in order to ensure the convergence of $\left(\boldsymbol{\sigma}_{h}, \mathbf{v}_{h}, \boldsymbol{\lambda}_{H}\right)$ to $(\boldsymbol{\sigma}, \mathbf{v}, \boldsymbol{\lambda})$.

\section{The fictitious domain method using the $\mathcal{Q}_{1}^{\text {div }}-\mathcal{Q}_{0}$ element.}

3.1. Position of the problem. For the volume unknowns, we introduce a regular mesh $\mathcal{T}_{h}$ of the rectangular domain $C$ composed of square elements of length $h$. In [5], the authors introduced for the problem without obstacle new mixed finite elements, the so-called $\mathcal{Q}_{k+1}^{\text {div }}-\mathcal{Q}_{k}$ elements, inspired by Nédélec's second family [32]. These elements, which are a generalization to elastodynamics of those presented in [6] for the scalar case, are compatible with mass lumping and therefore allow for constructing explicit schemes in time. A nonstandard convergence analysis of these $\mathcal{Q}_{k+1}^{\text {div }}-\mathcal{Q}_{k}$ elements has been carried out, showing the convergence without the fictitious domain method. Our first choice for the approximation spaces of the problem with an obstacle was naturally the lowest order element $\mathcal{Q}_{1}^{\text {div }}-\mathcal{Q}_{0}$ for the stress tensor and the velocity field

$$
\left\{\begin{array}{l}
\underline{\underline{X}}_{h}^{s y m}=\left\{\boldsymbol{\tau}_{h} \in \underline{\underline{X}}^{s y m} / \forall K \in \mathcal{T}_{h}, \tau_{h \mid K} \in\left(\mathcal{Q}_{1} \times \mathcal{Q}_{1}\right)^{2}\right\}, \\
\underline{M}_{h}=\underline{M}_{h}^{0}=\left\{\mathbf{w}_{h} \in \underline{M} / \mathbf{w}_{h_{K}} \in\left(Q_{0}\right)^{2}\right\} .
\end{array}\right.
$$

We point out that due to the symmetry of the stress tensor, another characterization of $\underline{\underline{X}}_{h}^{s y m}$ is

$$
\left\{\begin{aligned}
\underline{\underline{X}}_{h}^{\text {sym }}= & \left\{\boldsymbol{\tau}_{1,2} \in H^{1}(C) / \boldsymbol{\tau}_{1,2 \mid K} \in Q_{1} \forall K \in \mathcal{T}_{h}\right. \text { and } \\
& \left.\left(\boldsymbol{\tau}_{1,1}, \boldsymbol{\tau}_{2,2}\right) \in H(\operatorname{div}, C) /\left(\boldsymbol{\tau}_{1,1}, \boldsymbol{\tau}_{2,2}\right)_{\mid K} \in\left(Q_{1}\right)^{2} \forall K \in \mathcal{T}_{h}\right\} .
\end{aligned}\right.
$$

The degrees of freedom of the lowest order element are illustrated in Figure 3.1. We recall here the approximation properties for the space ${\underline{X_{h}}}^{\text {sym }}$. Let $\tau \in \underline{\underline{X}}^{\text {sym }}$ with 


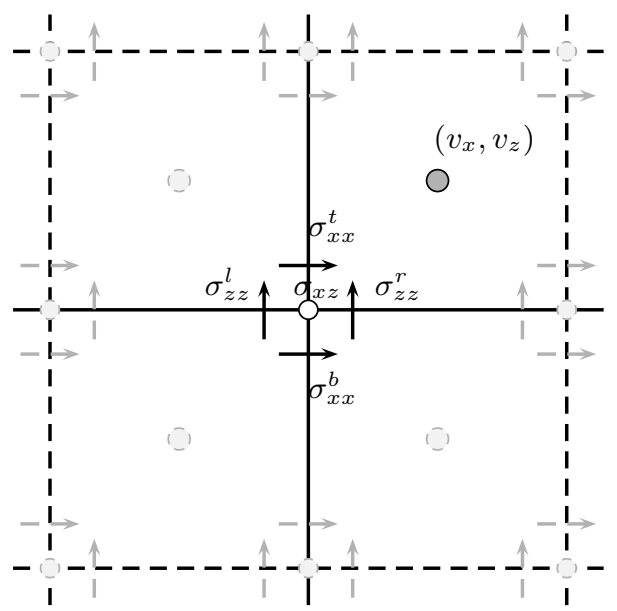

FIG. 3.1. Degrees of freedom for the mixed finite element defined by (3.1).

$\left(\tau_{11}, \tau_{22}\right) \in H^{1,0} \times H^{0,1}$ (see [9] for the definition of these spaces) and $\tau_{12}=\tau_{21} \in H^{1}$; then

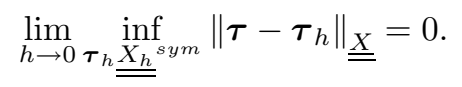

Moreover, if $\left(\tau_{11}, \tau_{22}\right) \in H^{2,1} \times H^{1,2}$ and $\tau_{12} \in H^{2}$, then

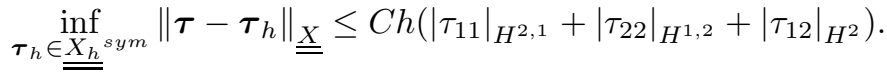

For the approximation of the Lagrange multiplier, we introduce a mesh of $\Gamma$ composed by $N$ curvilinear segments $S_{j}$ of length $H_{j}$, and we set $H=\sup _{j} H_{j}$. We assume that this mesh is uniformly regular

$$
\exists \nu, 0<\nu \leq 1 \text { such that: } \forall j, 1 \leq j \leq N, H_{j} \geq \nu H \text {. }
$$

We then choose the space of continuous linear piecewise functions:

$$
\underline{\mathcal{G}}_{H}=\left\{\nu_{h} \in \underline{\mathcal{G}} / \forall S_{j}, j=1, \ldots, N ; \nu_{H \mid S_{j}} \in\left(P_{1}\right)^{2}\right\} .
$$

The spaces $\left(\underline{\underline{X}}_{h}, \underline{M}_{h}^{0}, \underline{\mathcal{G}}_{H}\right)$ clearly satisfy the approximation properties (2.9).

This choice which seemed to us natural, since the convergence was proven without obstacle, is the one that was used in [8]. However, we have not been able to prove the convergence of the fictitious domain method with these spaces.

The convergence analysis of the fictitious domain method applied to other problems $[3,18,29]$ shows that convergence holds if a compatibility condition between the step sizes of the two meshes is satisfied,

$$
H \geq \alpha h .
$$

We will show in what follows some numerical illustrations which seem to indicate that for some special configurations of obstacles, the method does not converge. 
Before showing these numerical results, let us briefly recall the main difficulty of the convergence analysis in the case without an object. Introducing the linear operators

$$
\begin{aligned}
& B: \quad \underline{\underline{X}} \quad \longrightarrow \quad \underline{M}^{\prime} \\
& \boldsymbol{\sigma} \mapsto B(\boldsymbol{\sigma}): \quad \underline{M} \quad \longrightarrow \quad \mathbb{R} \\
& \mathbf{w} \quad \mapsto \quad\langle B(\boldsymbol{\sigma}), \mathbf{w}\rangle=b(\boldsymbol{\sigma}, \mathbf{w}), \\
& B_{h}: \underline{\underline{X}}_{h} \quad \longrightarrow \quad \underline{M}_{h}^{\prime} \\
& \boldsymbol{\sigma}_{h} \mapsto B_{h}\left(\boldsymbol{\sigma}_{h}\right): \underline{M}_{h} \quad \longrightarrow \mathbb{R} \\
& \mathbf{w}_{h} \mapsto\left\langle B_{h}\left(\boldsymbol{\sigma}_{h}\right), \mathbf{w}_{h}\right\rangle=b\left(\boldsymbol{\sigma}_{h}, \mathbf{w}_{h}\right),
\end{aligned}
$$

it is easy to verify that the inclusion

$$
\operatorname{Ker}\left(B_{h}\right) \subset \operatorname{Ker}(B)
$$

is not satisfied and that furthermore the bilinear form $a(.,$.$) is not coercive on \operatorname{Ker}\left(B_{h}\right)$ (even if it is on $\operatorname{Ker}(B)$ ), so that our problem does not fit the classical mixed finite element theory (cf. $[13,19]$ ). It was, however, possible to overcome this difficulty when dealing with the problem without the fictitious domain method. When coupled with the fictitious domain method, the same technique cannot be applied.

3.2. Numerical illustrations. We present in what follows some numerical results that illustrate the difficulties related to the convergence of the method that we discussed in the previous section.

The computational domain is the square $[0,10] \times[0,10] \mathrm{mm}^{2}$ composed by a homogeneous isotropic material with density and Lamé coefficients given by

$$
\rho=1000 \mathrm{Kgr} / \mathrm{m}^{3}, \quad \lambda=3.45 \times 10^{9} \mathrm{~Pa}, \quad \mu=2.04 \times 10^{9} \mathrm{~Pa} .
$$

We introduce an initial condition on the velocity field centered on $\left(x_{c}, z_{c}\right)=(5,5)$ $\mathrm{mm}$,

$$
\mathbf{v}((x, z), t=0)=0.1 F\left(\frac{r}{r_{0}}\right) \frac{\mathbf{r}}{r},
$$

where $F(r)$ is supported in $[0,1]$ and given by (for $r \in[0,1]$ )

$$
F(r)=A_{0}-A_{1} \cos (2 \pi r)+A_{2} \cos (3 \pi r)-A_{3} \cos (6 \pi r),
$$

with $\mathbf{r}=\left(x-x_{c}, z-z_{c}\right)^{t}, r=\|\mathbf{r}\|, r_{0}=1.5 \mathrm{~mm}$, and

$$
A_{0}=0.35875, \quad A_{1}=0.48829, \quad A_{2}=0.14128, \quad A_{3}=0.01168 \text {. }
$$

We consider a uniform mesh of squares using a discretization step $h=0.025 \mathrm{~mm}$. The time discretization is done using a leapfrog scheme with the time step $\Delta t$ chosen in such a way that the ratio $\Delta t / h$ is equal to the maximal value that ensures the stability. Perfectly matched layers $[11,16]$ are used to simulate a nonbounded domain in all of the boundaries. 


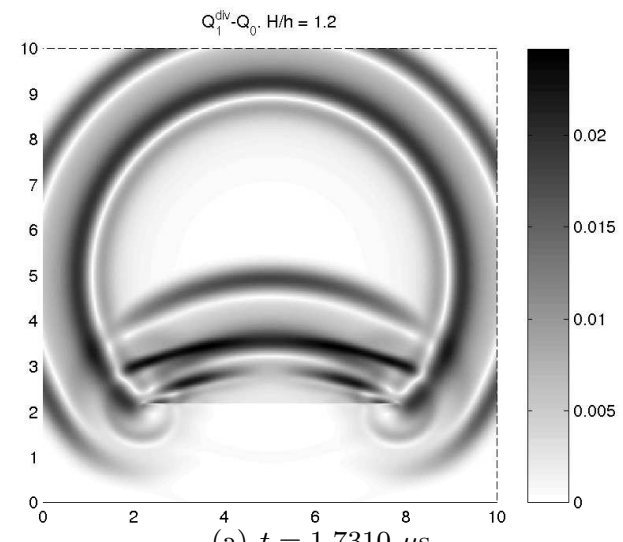

(a) $t=\stackrel{6}{1} .7310^{8} \mu \mathrm{s}$

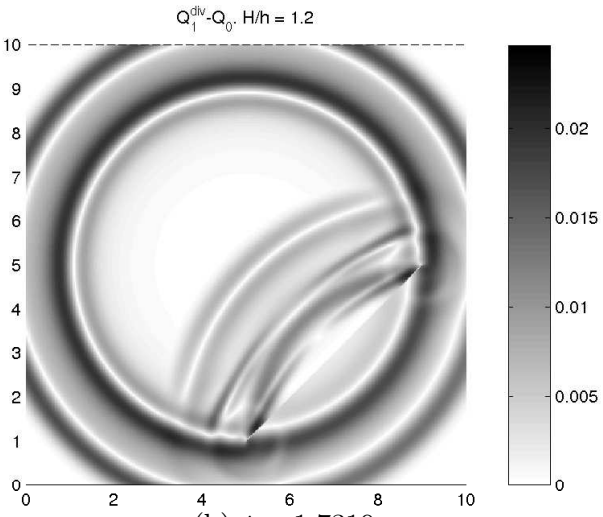

(b) $t={ }^{6} 1.7310^{8} \mu \mathrm{s}$

FIG. 3.2. $Q_{1}^{\text {div }}-Q_{0}$. Isotropic medium. Modulus of the velocity field. $H / h=1.2$.

Horizontal obstacle. In the first experiment we consider a plane horizontal crack

$$
(x, z)=(5+2 \sqrt{2}(2 t-1), 5-2 \sqrt{2}) \mathrm{mm}, \quad t \in[0,1],
$$

that we discretize using a uniform mesh of step $H=R h$. The method converges, and we obtain good results for reasonable values for the parameter $R$ (in the interval $[0.75,3])$. In Figure 3.2(a) we show a snapshot of the modulus of the velocity field for $R=1.2$. We can see the incident pressure wave that has reached the boundary of the computational domain, the reflected waves, and the scattered waves created by the two tips of the crack.

Diagonal obstacle. In the second experiment we treat a plane diagonal defect given by

$$
(x, z)=(5+4 t, 1+4 t) \mathrm{mm}, \quad t \in[0,1],
$$

that is, the same obstacle considered in the previous paragraph rotated by $\pi / 4$ radians with respect to $\left(x_{c}, z_{c}\right)$, the center of the initial condition. We point out that the initial condition satisfies

$$
v((x, z), t=0)=Q v((\tilde{x}, \tilde{z}), t=0),
$$

with

$$
(\tilde{x}, \tilde{z})^{t}=Q^{t}(x, z)^{t}+\left(x_{c}, z_{c}\right)^{t}, \quad Q=\left(\begin{array}{cc}
\cos \left(\frac{\pi}{4}\right) & \sin \left(\frac{\pi}{4}\right) \\
-\sin \left(\frac{\pi}{4}\right) & \cos \left(\frac{\pi}{4}\right)
\end{array}\right) .
$$

As the domain is isotropic, the velocity field obtained with the diagonal crack, denoted by $\mathbf{v}_{\text {diag }}$, and the one obtained with the horizontal one, denoted by $\mathbf{v}_{\text {hor }}$, satisfy

$$
\mathbf{v}_{\text {hor }}(x, z)=Q \mathbf{v}_{\text {diag }}(\tilde{x}, \tilde{z}) \text { and }\left|\mathbf{v}_{\text {hor }}\right|(x, z)=\left|\mathbf{v}_{\text {diag }}\right|(\tilde{x}, \tilde{z}) .
$$

In the same way as in the horizontal case, we discretize the Lagrange multiplier using a uniform grid of step $H=R h$, with several values for the parameter $R$. However, this time, the approximated solution does not seem to converge towards the physical solution (see, for instance, Figure 3.2(b) for $R=1.2$ ). The incident wave is not completely reflected but also transmitted through the interface. The scattered waves created by the tips of the crack are also poorly approximated. 


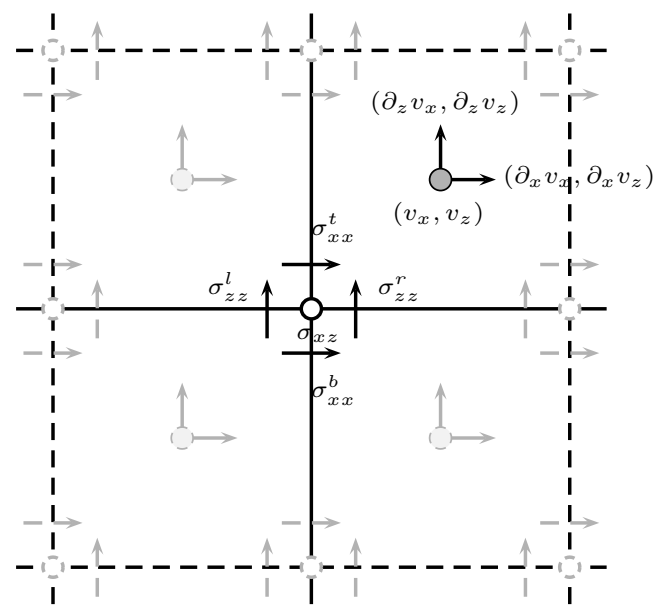

FIG. 4.1. Degrees of freedom of the modified mixed finite element $Q_{1}^{\text {div }} \times P_{1}^{\text {disc }}$.

\section{The modified element $Q_{1}^{\text {div }}-P_{1}^{\text {disc }}$.}

4.1. Presentation of the modified element. As explained in section 3.1, one of the difficulties of the convergence analysis of the $Q_{1}^{\text {div }} \times P_{1}^{\text {disc }}$, even in the absence of an obstacle, was the fact that inclusion (3.7) is not satisfied. In order to avoid this problem, and following the same ideas the authors introduced in [10], we propose to modify the space $M_{h}$ in such a way that

$$
\operatorname{div}\left(\underline{\underline{X}}_{h}^{\text {sym }}\right) \subset \underline{M}_{h},
$$

which easily implies (3.7) and could get rid of the lack of convergence observed in section 3.2 and simplify the analysis. That is why we have chosen to discretize the pressure in the space

$$
\underline{M}_{h}=\underline{M}_{h}^{1} \text { with } \underline{M}_{h}^{1}=\left\{\mathbf{w}_{h} \in \underline{M} / \forall K \in \mathcal{T}_{h}, \mathbf{w}_{h \mid K} \in\left(P_{1}(K)\right)^{2}\right\} .
$$

With this enrichment of the approximation space, we will have six degrees of freedom per element on the unknown $\mathbf{v}_{h}$ as shown in Figure 4.1. Since $\underline{M}_{h}^{0} \subset \underline{M}_{h}^{1}$ we have obviously

$$
\inf _{\mathbf{w}_{h} \in \underline{M}_{h}}\left\|\mathbf{v}-\mathbf{w}_{h}\right\|_{\rho} \leq \inf _{\mathbf{w}_{h}^{0} \in \underline{M}_{h}^{0}}\left\|\mathbf{v}-\mathbf{w}_{h}^{0}\right\|_{\rho},
$$

and, in consequence, the approximation properties (2.9) are still satisfied.

Remark 2. Assuming (4.1) and that the density is constant on each element, we have that

$$
\boldsymbol{\tau}_{h} \in \underline{\underline{X}}_{h} \Longrightarrow \mathbf{w}_{h}:=\frac{1}{\rho} \operatorname{div}\left(\boldsymbol{\tau}_{h}\right) \in \underline{M}_{h} .
$$

Introducing this particular test function in the second equation of (2.10), we obtain

$$
\frac{\mathrm{d}}{\mathrm{d} t} \int_{C} \mathbf{v}_{h} \cdot \operatorname{div}\left(\boldsymbol{\tau}_{h}\right)-\int_{C} \frac{1}{\rho} \operatorname{div}\left(\boldsymbol{\sigma}_{h}\right) \cdot \operatorname{div}\left(\boldsymbol{\tau}_{h}\right)=\int_{C} \frac{1}{\rho} \mathbf{f} \cdot \operatorname{div}\left(\boldsymbol{\tau}_{h}\right) .
$$


Deriving with respect to time the first and third equations of (2.10) and using the last expression, we deduce that our variational formulation implies the following second order formulation:

$$
\left\{\begin{array}{l}
\text { Find }\left(\boldsymbol{\sigma}_{h}(t), \tilde{\boldsymbol{\lambda}}_{H}(t)\right) \in \underline{\underline{X}}_{h} \times \underline{\mathcal{G}}_{H} \text { such that } \forall\left(\boldsymbol{\tau}_{h}, \boldsymbol{\mu}_{H}\right) \in \underline{\underline{X}}_{h} \times \underline{\mathcal{G}}_{H} \\
\frac{\mathrm{d}^{2}}{\mathrm{~d} t^{2}} \int_{C} \mathbf{A} \boldsymbol{\sigma}_{h}: \boldsymbol{\tau}_{h}+\int_{C} \frac{1}{\rho} \operatorname{div}\left(\boldsymbol{\tau}_{h}\right) \cdot \operatorname{div}\left(\boldsymbol{\sigma}_{h}\right)-\int_{\Gamma} \boldsymbol{\tau}_{h} \mathbf{n} \cdot \tilde{\boldsymbol{\lambda}}_{H}=\int_{C}-\frac{1}{\rho} f \cdot \operatorname{div}\left(\boldsymbol{\tau}_{h}\right) \\
\int_{\Gamma} \boldsymbol{\sigma}_{h} \mathbf{n} \cdot \boldsymbol{\mu}_{H}=0, \\
\boldsymbol{\sigma}_{h}(t=0)=\boldsymbol{\sigma}_{h, 0}, \quad \frac{\partial \boldsymbol{\sigma}_{h}}{\partial t}(t=0)=\mathbf{C} \varepsilon\left(\mathbf{v}_{h, 0}\right)
\end{array}\right.
$$

where $\tilde{\boldsymbol{\lambda}}_{H}=\frac{\partial \boldsymbol{\lambda}_{H}}{\partial t}$. The nature of this problem is close to the ones analyzed in $[18,29]$.

4.2. Some comments about the convergence of the modified element. From the numerical point of view we observe that the method converges under a compatibility condition of the form (3.6) between the two meshes. In section 4.5, repeating the numerical experiment of section 3.2 using the $Q_{1}^{\text {div }} \times P_{1}^{\text {disc }}$ element, we observe that the results are similar regardless of the orientation of the crack, concluding that the method converges. In section 5 we compute numerically the order of convergence of the new method for a particular case.

From the theoretical point of view the convergence proof in this case is not a straightforward generalization of the result obtained in [10] for the scalar case. The main difficulty comes from the nonstandard regularity required to obtain the approximation properties for $\underline{\underline{X}}_{h}^{\text {sym }}$ (see (3.3)). Indeed, the maximal regularity (in space) of the stress tensor in the case of a domain with a crack is $\sigma \in\left(H^{\frac{1}{2}-\varepsilon}(\operatorname{div}, C)\right)^{2}$, and $\boldsymbol{\sigma}$ is symmetric. This regularity is not sufficient to obtain (3.3), and thus we cannot conclude (see [10] for more details on the differences with the scalar case). However, guided by the results obtained for the scalar case, we conjecture the following result.

Conjecture 4.1. Let $\mathbf{f} \in C^{0}([0, T], \underline{M}), \boldsymbol{\sigma}_{0} \in \underline{X}^{\text {sym }}, \mathbf{v}_{0} \in \underline{M}$ satisfying (2.3) and $(\boldsymbol{\sigma}, \mathbf{v}, \boldsymbol{\lambda})$ be the solution of the problem $(2.6)$. $\overline{\overline{\text { Let }}}\left(\boldsymbol{\sigma}_{h}, \mathbf{v}_{h}, \overline{\left.\boldsymbol{\lambda}_{H}\right)}\right.$ be the solution of (2.10) with initial data $\left(\boldsymbol{\sigma}_{h, 0}, \mathbf{v}_{h, 0}\right)$ that are first order approximations of $\left(\boldsymbol{\sigma}_{0}, \mathbf{v}_{0}\right)$, and assume that $(3.6) r$ is satisfied. Then if $(\boldsymbol{\sigma}, \mathbf{v}, \boldsymbol{\lambda}) \in C^{2}\left([0, T],\left(H^{\frac{1}{2}-\varepsilon}(\operatorname{div}, C)\right)^{2} \times\right.$ $\left.\left(H^{\frac{1}{2}-\varepsilon}(C)\right)^{2} \times\left(H^{1-\varepsilon}(\Gamma)\right)^{2}\right)$,

$$
\begin{gathered}
\left\|\boldsymbol{\sigma}_{h}-\boldsymbol{\sigma}\right\|_{C^{0}\left([0, T] ; \underline{\underline{X}}^{s y m}\right)}+\left\|\mathbf{v}_{h}-\mathbf{v}\right\|_{C^{0}([0, T] ; \underline{M})}+\left\|\boldsymbol{\lambda}_{H}-\boldsymbol{\lambda}\right\|_{C^{0}([0, T] ; \underline{\mathcal{G}})} \leq \\
\mathcal{C}\left(h^{\frac{1}{2}-\varepsilon}+H^{\frac{1}{2}-\varepsilon}\right)(1+T)\left(\|\boldsymbol{\sigma}\|_{C^{2}\left([0, T] ;\left(H^{\frac{1}{2}-\varepsilon}(\operatorname{div}, C)\right)^{2}\right)}+\right. \\
\left.\|\mathbf{v}\|_{C^{2}\left([0, T] ;\left(H^{\frac{1}{2}-\varepsilon}(C)\right)^{2}\right)}+\|\boldsymbol{\lambda}\|_{C^{2}\left([0, T] ;\left(H^{1-\varepsilon}(\Gamma)\right)^{2}\right)}\right) .
\end{gathered}
$$

The numerical results obtained in section 5 confirm this result.

4.3. Dispersion analysis of the modified element. It is useful and classical to perform a dispersion analysis in order to specify the properties of a discrete scheme (in the absence of an obstacle) (e.g., [36, 14]). This consists in studying the behavior of discrete plane waves propagated with the scheme. We will show that four spurious modes that contaminate the discrete solution appear, for the modified element, because four new degrees of freedom per element were added to the velocity field. 
Assume that our computational domain $\Omega=\mathbb{R}^{2}$ is homogeneous and that we use a uniform mesh composed by squares of edge $h$. In this way, the degrees of freedom of the stress tensor are placed at the vertices of the squares, that is, at the points $\left(x_{i}, z_{j}\right)=(i h, j h)$. We thus have

$$
\left(\sigma_{x, x}^{t}\right)_{i, j}, \quad\left(\sigma_{x, x}^{b}\right)_{i, j}, \quad\left(\sigma_{z, z}^{r}\right)_{i, j}, \quad\left(\sigma_{z, z}^{l}\right)_{i, j}, \quad\left(\sigma_{x, z}\right)_{i, j}, \quad(i, j) \in \mathbb{Z} \times \mathbb{Z} .
$$

The degrees of freedom for the velocity field are defined at the center of each element, that is, at the points $\left(x_{i+\frac{1}{2}}, z_{j+\frac{1}{2}}\right)=((i+1 / 2) h,(j+1 / 2) h)$. The corresponding unknowns are denoted by

$$
\begin{array}{llll}
\left(v_{x}\right)_{i+\frac{1}{2}, j+\frac{1}{2}}, & \left(\partial_{x} v_{x}\right)_{i+\frac{1}{2}, j+\frac{1}{2}}, & \left(\partial_{z} v_{x}\right)_{i+\frac{1}{2}, j+\frac{1}{2}}, & (i, j) \in \mathbb{Z} \times \mathbb{Z}, \\
\left(v_{z}\right)_{i+\frac{1}{2}, j+\frac{1}{2}}, & \left(\partial_{x} v_{z}\right)_{i+\frac{1}{2}, j+\frac{1}{2}}, & \left(\partial_{z} v_{z}\right)_{i+\frac{1}{2}, j+\frac{1}{2}}, & (i, j) \in \mathbb{Z} \times \mathbb{Z} .
\end{array}
$$

The numerical scheme is thus given by

$$
\begin{aligned}
& \begin{aligned}
\frac{\mathrm{d}}{\mathrm{d} t}\left(v_{x}\right)_{i+\frac{1}{2}, j+\frac{1}{2}}= & \frac{1}{2 \rho h}\left\{\left(\sigma_{x x}^{t}\right)_{i+1, j}-\left(\sigma_{x x}^{t}\right)_{i, j}+\left(\sigma_{x x}^{b}\right)_{i+1, j+1}-\left(\sigma_{x x}^{b}\right)_{i, j+1}\right. \\
& \left.+\left(\sigma_{x z}\right)_{i+1, j+1}-\left(\sigma_{x z}\right)_{i+1, j}+\left(\sigma_{x z}\right)_{i, j+1}-\left(\sigma_{x z}\right)_{i, j}\right\}, \\
\frac{\mathrm{d}}{\mathrm{d} t}\left(\partial_{x} v_{x}\right)_{i+\frac{1}{2}, j+\frac{1}{2}}= & \frac{1}{\sqrt{12} \rho h}\left\{\left(\sigma_{x z}\right)_{i+1, j+1}+\left(\sigma_{x z}\right)_{i, j}-\left(\sigma_{x z}\right)_{i+1, j}-\left(\sigma_{x z}\right)_{i, j+1}\right\}, \\
\frac{\mathrm{d}}{\mathrm{d} t}\left(\partial_{z} v_{x}\right)_{i+\frac{1}{2}, j+\frac{1}{2}}= & \frac{1}{\sqrt{12} \rho h}\left\{\left(\sigma_{x x}^{b}\right)_{i+1, j+1}+\left(\sigma_{x x}^{t}\right)_{i, j}-\left(\sigma_{x x}^{t}\right)_{i+1, j}-\left(\sigma_{x x}^{b}\right)_{i, j+1}\right\},
\end{aligned} \\
& \begin{aligned}
\frac{\mathrm{d}}{\mathrm{d} t}\left(v_{z}\right)_{i+\frac{1}{2}, j+\frac{1}{2}}= & \frac{1}{2 \rho h}\left\{\left(\sigma_{x z}\right)_{i+1, j}-\left(\sigma_{x z}\right)_{i, j}+\left(\sigma_{x z}\right)_{i+1, j+1}-\left(\sigma_{x z}\right)_{i, j+1}\right. \\
& \left.+\left(\sigma_{z z}^{r}\right)_{i, j+1}-\left(\sigma_{z z}^{r}\right)_{i, j}+\left(\sigma_{z z}^{l}\right)_{i+1, j+1}-\left(\sigma_{z z}^{l}\right)_{i+1, j}\right\} \\
\frac{\mathrm{d}}{\mathrm{d} t}\left(\partial_{x} v_{z}\right)_{i+\frac{1}{2}, j+\frac{1}{2}}= & \frac{1}{\sqrt{12} \rho h}\left\{\left(\sigma_{z z}^{l}\right)_{i+1, j+1}+\left(\sigma_{z z}^{r}\right)_{i, j}-\left(\sigma_{z z}^{l}\right)_{i+1, j}-\left(\sigma_{z z}^{r}\right)_{i, j+1}\right\} \\
\frac{\mathrm{d}}{\mathrm{d} t}\left(\partial_{z} v_{z}\right)_{i+\frac{1}{2}, j+\frac{1}{2}}= & \frac{1}{\sqrt{12} \rho h}\left\{\left(\sigma_{x z}\right)_{i+1, j+1}+\left(\sigma_{x z}\right)_{i, j}-\left(\sigma_{x z}\right)_{i+1, j}-\left(\sigma_{x z}\right)_{i, j+1}\right\}
\end{aligned}
\end{aligned}
$$$$
\left[\begin{array}{ccccc}
\frac{A_{1,1}}{2} & 0 & \frac{A_{1,2}}{4} & \frac{A_{1,2}}{4} & \frac{A_{1,3}}{2} \\
0 & \frac{A_{1,1}}{2} & \frac{A_{1,2}}{4} & \frac{A_{1,2}}{4} & \frac{A_{1,3}}{2} \\
\frac{A_{1,2}}{4} & \frac{A_{1,2}}{4} & \frac{A_{2,2}}{2} & 0 & \frac{A_{2,3}}{2} \\
\frac{A_{1,2}}{4} & \frac{A_{1,2}}{4} & 0 & \frac{A_{2,2}}{2} & \frac{A_{2,3}}{2} \\
\frac{A_{1,3}}{2} & \frac{A_{1,3}}{2} & \frac{A_{2,3}}{2} & \frac{A_{2,3}}{2} & A_{3,3}
\end{array}\right]\left[\begin{array}{c}
\frac{\mathrm{d}}{\mathrm{d} t}\left(\sigma_{x x}^{t}\right)_{i, j} \\
\frac{\mathrm{d}}{\mathrm{d} t}\left(\sigma_{x x}^{b}\right)_{i, j} \\
\frac{\mathrm{d}}{\mathrm{d} t}\left(\sigma_{z z}^{r}\right)_{i, j} \\
\frac{\mathrm{d}}{\mathrm{d} t}\left(\sigma_{z z}^{l}\right)_{i, j} \\
\frac{\mathrm{d}}{\mathrm{d} t}\left(\sigma_{x z}\right)_{i, j}
\end{array}\right]=\left[\begin{array}{c}
\left(B_{1}\right)_{i, j} \\
\left(B_{2}\right)_{i, j} \\
\left(B_{3}\right)_{i, j} \\
\left(B_{4}\right)_{i, j} \\
\left(B_{5}\right)_{i, j}
\end{array}\right]
$$ 
with

$$
\mid \begin{aligned}
\left(B_{1}\right)_{i, j}= & \frac{1}{2 h}\left(\left(v_{x}\right)_{i+\frac{1}{2}, j+\frac{1}{2}}-\left(v_{x}\right)_{i-\frac{1}{2}, j+\frac{1}{2}}-\frac{1}{\sqrt{3}}\left(\left(\partial_{z} v_{x}\right)_{i+\frac{1}{2}, j+\frac{1}{2}}-\left(\partial_{z} v_{x}\right)_{i-\frac{1}{2}, j+\frac{1}{2}}\right)\right) \\
\left(B_{2}\right)_{i, j}= & \frac{1}{2 h}\left(\left(v_{x}\right)_{i+\frac{1}{2}, j-\frac{1}{2}}-\left(v_{x}\right)_{i-\frac{1}{2}, j-\frac{1}{2}}+\frac{1}{\sqrt{3}}\left(\left(\partial_{z} v_{x}\right)_{i+\frac{1}{2}, j-\frac{1}{2}}-\left(\partial_{z} v_{x}\right)_{i-\frac{1}{2}, j-\frac{1}{2}}\right)\right) \\
\left(B_{3}\right)_{i, j}= & \frac{1}{2 h}\left(\left(v_{z}\right)_{i+\frac{1}{2}, j+\frac{1}{2}}-\left(v_{z}\right)_{i+\frac{1}{2}, j-\frac{1}{2}}-\frac{1}{\sqrt{3}}\left(\left(\partial_{x} v_{z}\right)_{i+\frac{1}{2}, j+\frac{1}{2}}-\left(\partial_{x} v_{z}\right)_{i+\frac{1}{2}, j-\frac{1}{2}}\right)\right) \\
\left(B_{4}\right)_{i, j}= & \frac{1}{2 h}\left(\left(v_{z}\right)_{i-\frac{1}{2}, j+\frac{1}{2}}-\left(v_{z}\right)_{i-\frac{1}{2}, j-\frac{1}{2}}+\frac{1}{\sqrt{3}}\left(\left(\partial_{x} v_{z}\right)_{i-\frac{1}{2}, j+\frac{1}{2}}-\left(\partial_{x} v_{z}\right)_{i-\frac{1}{2}, j-\frac{1}{2}}\right)\right) \\
\left(B_{5}\right)_{i, j}= & \frac{1}{2 h}\left(\left(v_{x}\right)_{i+\frac{1}{2}, j+\frac{1}{2}}-\left(v_{x}\right)_{i+\frac{1}{2}, j-\frac{1}{2}}+\left(v_{x}\right)_{i-\frac{1}{2}, j+\frac{1}{2}}-\left(v_{x}\right)_{i-\frac{1}{2}, j-\frac{1}{2}}\right) \\
& +\frac{1}{2 h}\left(\left(v_{z}\right)_{i+\frac{1}{2}, j+\frac{1}{2}}-\left(v_{z}\right)_{i-\frac{1}{2}, j+\frac{1}{2}}+\left(v_{z}\right)_{i+\frac{1}{2}, j-\frac{1}{2}}-\left(v_{z}\right)_{i-\frac{1}{2}, j-\frac{1}{2}}\right) \\
& +\frac{1}{\sqrt{12} h}\left(\left(\partial_{x} v_{x}\right)_{i+\frac{1}{2}, j-\frac{1}{2}}-\left(\partial_{x} v_{x}\right)_{i+\frac{1}{2}, j+\frac{1}{2}}+\left(\partial_{x} v_{x}\right)_{i-\frac{1}{2}, j+\frac{1}{2}}-\left(\partial_{x} v_{x}\right)_{i-\frac{1}{2}, j-\frac{1}{2}}\right) \\
& +\frac{1}{\sqrt{12} h}\left(\left(\partial_{z} v_{z}\right)_{i-\frac{1}{2}, j+\frac{1}{2}}-\left(\partial_{z} v_{z}\right)_{i+\frac{1}{2}, j+\frac{1}{2}}+\left(\partial_{z} v_{z}\right)_{i+\frac{1}{2}, j-\frac{1}{2}}-\left(\partial_{z} v_{z}\right)_{i-\frac{1}{2}, j-\frac{1}{2}}\right)
\end{aligned} .
$$

For the sake of simplicity we treat the isotropic case; that is, we assume that

$$
\left(\begin{array}{ccc}
A_{1,1} & A_{1,2} & A_{1,3} \\
A_{1,2} & A_{2,2} & A_{2,3} \\
A_{1,3} & A_{2,3} & A_{3,3}
\end{array}\right)=\left(\begin{array}{ccc}
\frac{2 \mu+\lambda}{4 \mu(\lambda+\mu)} & -\frac{\lambda}{4 \mu(\lambda+\mu)} & 0 \\
-\frac{\lambda}{4 \mu(\lambda+\mu)} & \frac{2 \mu+\lambda}{4 \mu(\lambda+\mu)} & 0 \\
0 & 0 & \frac{1}{\mu}
\end{array}\right)
$$

where $\lambda$ and $\mu$ are the Lamé coefficients of the material. The next step is to eliminate the unknowns associated to the stress tensor and to consider a plane wave with frequency $\omega$ and wave vector $\boldsymbol{k}=\left(k_{x}, k_{z}\right)=k(\cos \theta, \sin \theta)$ :

$$
\begin{gathered}
\left(\begin{array}{c}
\left(v_{x}\right)_{i+\frac{1}{2}, j+\frac{1}{2}} \\
\left(v_{z}\right)_{i+\frac{1}{2}, j+\frac{1}{2}} \\
\left(\partial_{x} v_{x}\right)_{i+\frac{1}{2}, j+\frac{1}{2}} \\
\left(\partial_{z} v_{z}\right)_{i+\frac{1}{2}, j+\frac{1}{2}} \\
\left(\partial_{x} v_{z}\right)_{i+\frac{1}{2}, j+\frac{1}{2}} \\
\left(\partial_{z} v_{x}\right)_{i+\frac{1}{2}, j+\frac{1}{2}}
\end{array}\right)=\mathbf{V} \exp \left[i\left(c t-\left(\cos (\theta) x_{i+\frac{1}{2}}+\sin (\theta) z_{j+\frac{1}{2}}\right)\right)\right], \\
\mathbf{V}=\left[\mathbf{v}_{\mathbf{x}}, \mathbf{v}_{\mathbf{z}}, \partial_{\mathbf{x}} \mathbf{v}_{\mathbf{x}}, \partial_{\mathbf{z}} \mathbf{v}_{\mathbf{z}}, \partial_{\mathbf{x}} \mathbf{v}_{\mathbf{z}}, \partial_{\mathbf{z}} \mathbf{v}_{\mathbf{x}}\right]^{t},
\end{gathered}
$$

where $c=\omega / k$ is the phase velocity and $\mathbf{V}$ is the amplitude vector. Plugging this expression into the numerical scheme, we observe that the couple $\left(\omega^{2}, \boldsymbol{V}\right)$ has to satisfy the following eigenvalue problem:

$$
\mathcal{K}_{h} \boldsymbol{V}=\frac{\omega^{2}}{k^{2}} \boldsymbol{V}, \quad \text { with } k=|\boldsymbol{k}|,
$$

where the nonzero elements of the matrix $\mathcal{K}_{h}$ are given in Appendix A. 
The matrix $\mathcal{K}_{h}$ is Hermitian; thus, there are six real eigenvalues with six orthogonal eigenvectors. Performing a Taylor expansion we obtain the following results. For the two first couples denoted by $\left(\omega_{T}^{2}, \boldsymbol{V}_{T}\right), T \in\{P, S\}$, we have

$$
\begin{aligned}
c_{P}=\frac{\omega_{P}}{k} & =\sqrt{\frac{\lambda+2 \mu}{\rho}}+\mathcal{O}(k h)^{2}, \\
\mathbf{V}_{\mathbf{P}} & =[\cos \theta, \sin \theta, 0,0,0,0]^{t}+\mathcal{O}(k h), \\
c_{S}=\frac{\omega_{S}}{k} & =\sqrt{\frac{\mu}{\rho}}+\mathcal{O}(k h)^{2}, \\
\mathbf{V}_{\mathbf{S}} & =[-\sin \theta, \cos \theta, 0,0,0,0]^{t}+\mathcal{O}(k h) .
\end{aligned}
$$

The phase velocity of the these two waves is a second order approximation of the phase velocity of the continuous waves. The other four solutions of the eigenvalue problem are spurious modes produced by the introduction of the additional degrees of freedom and are given by

$$
\begin{aligned}
& \begin{aligned}
c_{\text {spur } 1}=\frac{\omega_{\text {spur } 1}}{k} & =\mathcal{O}(k h), \\
\mathbf{V}_{\text {spur1 }} & =[0,0,1,0,0,0]^{t}+\mathcal{O}(k h),
\end{aligned} \\
& \begin{aligned}
c_{\text {spur } 2}=\frac{\omega_{\text {spur } 2}}{k} & =\mathcal{O}(k h), \\
\mathbf{V}_{\text {spur2 }} & =[0,0,0,1,0,0]^{t}+\mathcal{O}(k h),
\end{aligned} \\
& \begin{aligned}
c_{\text {spur } 3}=\frac{\omega_{\text {spur } 3}}{k} & =\sqrt{\frac{4 \sin ^{2}(\theta)(\lambda+\mu) \mu}{3 \rho(\lambda+2 \mu)}}+\mathcal{O}(k h)^{2}, \\
\mathbf{V}_{\text {spur3 }} & =[0,0,0,0,1,0]^{t}+\mathcal{O}(k h),
\end{aligned} \\
& \begin{aligned}
c_{\text {spur } 4}=\frac{\omega_{\text {spur } 4}}{k} & =\sqrt{\frac{4 \cos ^{2}(\theta)(\lambda+\mu) \mu}{3 \rho(\lambda+2 \mu)}}+\mathcal{O}(k h)^{2}, \\
\mathbf{V}_{\text {spur4 }} & =[0,0,0,0,0,1]^{t}+\mathcal{O}(k h) .
\end{aligned}
\end{aligned}
$$

We remark that the first two spurious modes propagate with a phase velocity that goes to zero, and thus they are not really visible in the numerical simulations. The phase velocity of the other two modes is nonzero and always smaller than the shear wave velocity. Therefore, these spurious waves will propagate and pollute the physical solution.

4.4. Damping of the spurious modes. As we have seen in the previous section, the modified element gives rise to some spurious modes. In this section we propose a way to damp the amplitude of these modes (without damping the "physical" part of the solution), so that they do not pollute too much the approximate solution.

The approximate space $\underline{M}_{h}$ can be decomposed as

$$
\underline{M}_{h}=\underline{M}_{h}^{0} \oplus \underline{M}_{h}^{r},
$$

where $\underline{M}_{h}^{0}$ is the space of piecewise constants on each component and $\underline{M}_{h}^{r}$ is its orthogonal complement (for the $L^{2}$ scalar product). The space $\underline{M}_{h}^{r}$ is composed of $P^{1}$ discontinuous functions with a vanishing mean value per element and per component. 
From the dispersion analysis, we observe that the main components of the spurious modes (the $\mathcal{O}(1)$ part) belong to $\underline{M}_{h}^{r}$. In order to damp this main part, we introduce the $L^{2}$ orthogonal projection on $\underline{M}_{h}^{r}$, which we denote by $P_{M_{h}^{r}}$, defined for any $\mathbf{v}_{h} \in \underline{M}_{h}$ as

$$
P_{\underline{M}_{h}^{r}}\left(\mathbf{v}_{h}\right) \in \underline{M}_{h}^{r} \text { and }\left(P_{\underline{M}_{h}^{r}}\left(\mathbf{v}_{h}\right), \mathbf{w}_{h}\right)=\left(\mathbf{v}_{h}, \mathbf{w}_{h}\right) \quad \forall \mathbf{w}_{h} \in \underline{M}_{h}^{r} .
$$

The approximate problem with damping consists in finding $\left(\boldsymbol{\sigma}_{h}, \mathbf{v}_{h}, \boldsymbol{\lambda}_{H}\right) \in \underline{\underline{X}}_{h}^{s y m} \times$ $\underline{M}_{h} \times \underline{\mathcal{G}}_{H}$ such that

$$
\left\{\begin{array}{l}
\text { Find }\left(\boldsymbol{\sigma}_{h}(t), \mathbf{v}_{h}(t), \boldsymbol{\lambda}_{H}(t)\right) \in \underline{\underline{X}}_{h}^{s y m} \times \underline{M}_{h} \times \underline{\mathcal{G}}_{h} \text { satisfying } \\
\frac{\mathrm{d}}{\mathrm{d} t} a\left(\boldsymbol{\sigma}_{h}, \boldsymbol{\tau}_{h}\right)+b\left(\boldsymbol{\tau}_{h}, \mathbf{v}_{h}\right)-\left\langle\boldsymbol{\tau}_{h} \mathbf{n}, \boldsymbol{\lambda}_{H}\right\rangle_{\Gamma}=0 \quad \forall \boldsymbol{\tau}_{h} \in \underline{\underline{X}}_{h}^{s y m}, \\
\frac{\mathrm{d}}{\mathrm{d} t}\left(\mathbf{v}_{h}, \mathbf{w}_{h}\right)_{\rho}+\left(P_{\underline{M}_{h}^{r}}\left(\mathbf{v}_{h}\right), \mathbf{w}_{h}\right)_{\beta}-b\left(\boldsymbol{\sigma}_{h}, \mathbf{w}_{h}\right)=\left(\mathbf{f}, \mathbf{w}_{h}\right) \quad \forall \mathbf{w}_{h} \in \underline{M}_{h}, \\
\left\langle\boldsymbol{\sigma}_{h} \mathbf{n}, \boldsymbol{\mu}_{H}\right\rangle_{\Gamma}=0 \quad \forall \boldsymbol{\mu}_{h} \in \underline{\mathcal{G}}_{H} .
\end{array}\right.
$$

In this system $\beta$ represents a damping parameter, which is chosen as a positive constant in the applications. The case $\beta=0$ gives back the nondamped problem, while a strictly positive $\beta$ corresponds to a dissipative problem. In section 5 we will explain how to choose the value for this parameter.

4.5. Numerical illustrations using the modified element. Let us now show some numerical illustrations of the behavior of the fictitious domain method with the new finite element space. The numerical experiments that we have considered are the same as in section 3.2 and will allow us to compare both finite elements.

Horizontal obstacle. Once again we discretize the horizontal crack defined by (3.9) using a uniform mesh of step $H=R h$. The results obtained with the new mixed finite element $Q_{1}^{\text {div }}-P_{1}^{\text {disc }}$ are similar to those given by the $Q_{1}^{\text {div }}-Q_{0}$ element. The method converges for reasonable values of the parameter $R$ (in the interval $[0.75,3]$ ). In Figure 4.2 (a) we can see the results for $R=1.2$.

Diagonal obstacle. We now consider the diagonal crack defined by the expression (3.10). We recall that the continuous problem is a rotation of $\pi / 4$ radians with respect to the point $\left(x_{c}, z_{c}\right)=(5,5)$.

The Lagrange multiplier is again discretized using an uniform mesh of step $H=$ $R h$. Contrary to the results obtained with the element $Q_{1}^{\text {div }}-Q_{0}$, the ones given by the modified element $Q_{1}^{\text {div }}-P_{1}^{\text {disc }}$ converge towards the physical solution when choosing reasonable values for the ratio $H / h$. In Figure $4.2(\mathrm{~b})$, this time the incident wave is almost completely reflected by the obstacle. The scattered waves created by the tips of the crack are well approximated.

As explained before, the enrichment of the $\underline{M}_{h}$ approximation space introduces spurious modes in the solution. Although the amplitude of these nonphysical waves goes to zero with the size of the discretization step, it is still significant for a usual choice of the discretization parameters, typically corresponding to 20 points per wavelength. These spurious modes are, for example, visible in the results presented in Figure 4.3(a) where we have changed the scale to amplify the spurious phenomena.

In order to study in more detail these phenomena we represent in Figure 4.4 the evolution in time of the modulus of the velocity field at three points: $\mathbf{x}_{\mathbf{1}}=\left(x_{1}, z_{1}\right)=$ $(6.5,3.5) \mathrm{mm}, \mathbf{x}_{\mathbf{2}}=\left(x_{2}, z_{2}\right)=(7.5,2.5) \mathrm{mm}$, and $\mathbf{x}_{\mathbf{3}}=\left(x_{3}, z_{3}\right)=(5,0.5) \mathrm{mm}$. The first two points are centered with respect to the crack, one behind and the other in 


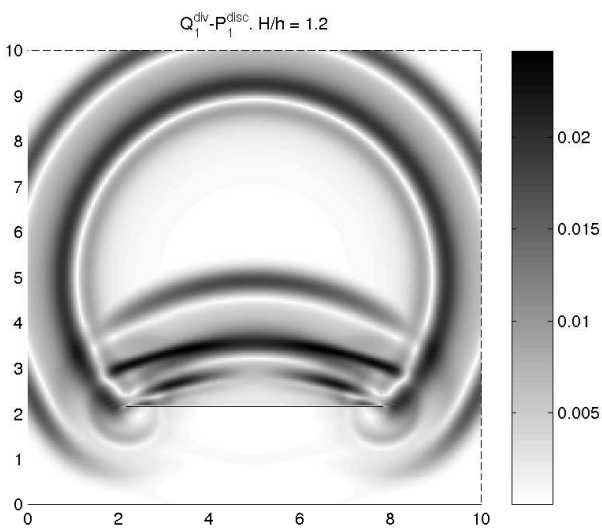

(a) $t=1.7310 \mu \mathrm{s}$

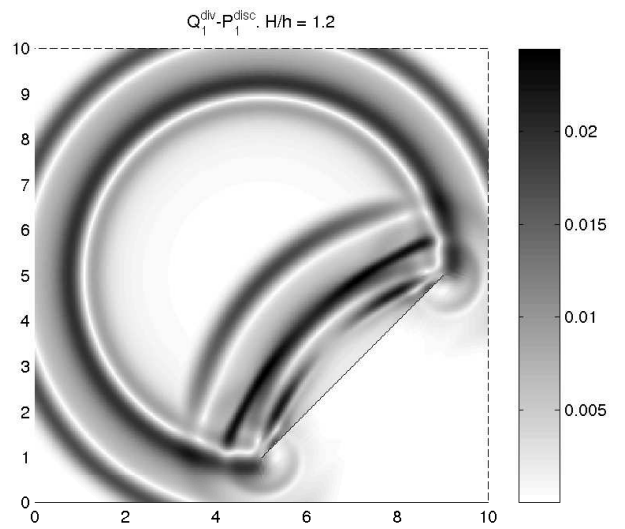

(b) $t=1.7310 \mu \mathrm{s}$

FIG. 4.2. $Q_{1}^{\text {div }}-P_{1}^{\text {disc }}$. Isotropic medium. Modulus of the velocity field. $H / h=1.2$.

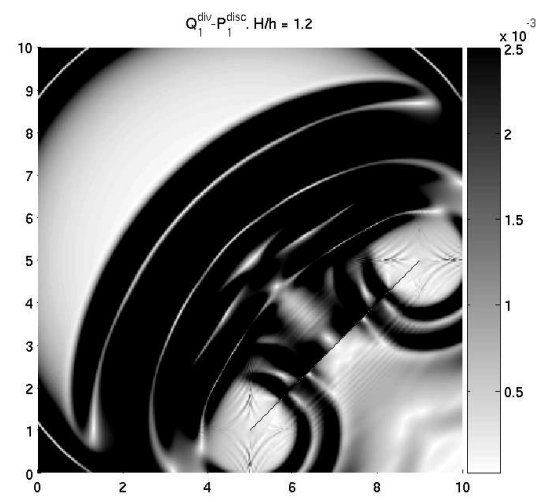

(a) $Q_{1}^{\text {div }}-P_{1}^{\text {disc }} \cdot \beta=0$

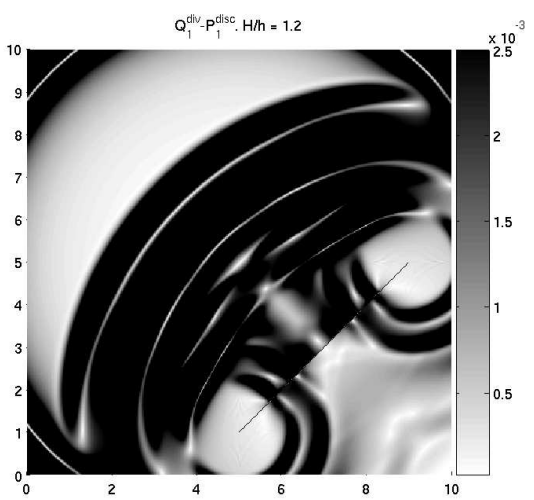

(b) $Q_{1}^{\text {div }}-P_{1}^{\text {disc }} \cdot \beta=5 \rho 10^{6}$

FIG. 4.3. Modulus of the velocity field at $t=2.5965 \mu \mathrm{s}$.

front of it. The third point is located near the lower tip of the crack, where the spurious waves seem stronger. To determine the speed of convergence of the method, we use three different meshes with a space discretization step of $h=0.025,0.0125$, and $0.00625 \mathrm{~mm}$, respectively.

The results obtained at the first point (in front of the crack) are already good with the coarse mesh (see Figure 4.4, top left). The amplitude of the spurious waves is very small with respect to the amplitude of the incident $(t \in[0.25,1] \mu \mathrm{s})$, reflected $(t \in[0.75,2] \mu \mathrm{s})$, and (first) scattered waves $(t \in[2.75,4.25] \mu \mathrm{s})$.

The second point is in the "shadow region," where the amplitude of the physical waves is about $15 \%$ smaller than the amplitude of the incident wave (see Figure 4.4, middle left). Consequently the error is more visible. In the time interval $t \in[0,2] \mu \mathrm{s}$ we can see the part of the incident wave that has been transmitted across the obstacle. The amplitude of those waves goes to zero when we refine the mesh. From $t=2$ to $t=4.5 \mu \mathrm{s}$ we can see the first group of scattered waves. At the beginning and at the end of this time interval we can see some oscillations that come from the spurious modes introduced by the enrichment of the velocity field discretization space. The 


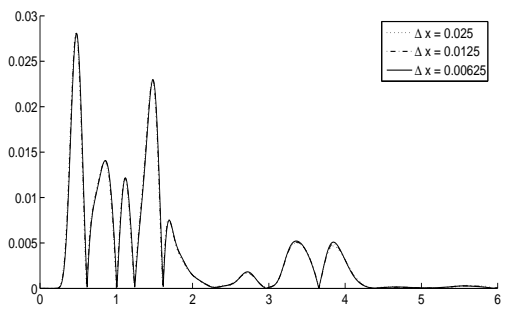

(a) $|v|((6.5,3.5) \mathrm{mm}, t), \beta=0$

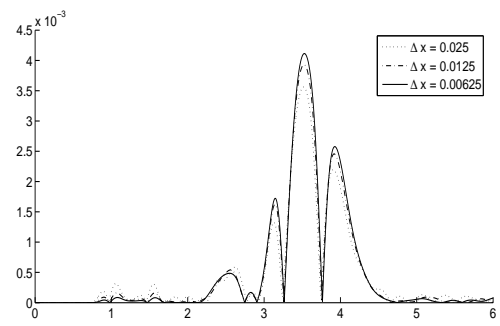

(c) $|v|((7.5,2.5) \mathrm{mm}, t), \beta=0$

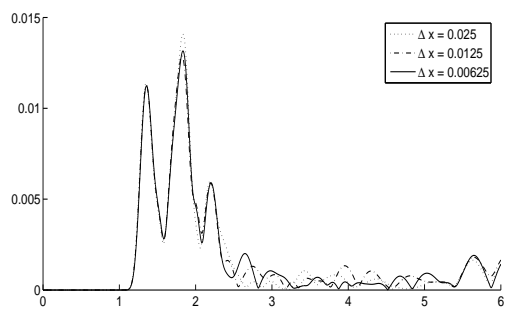

(e) $|v|((5.0,0.5), t), \beta=0$

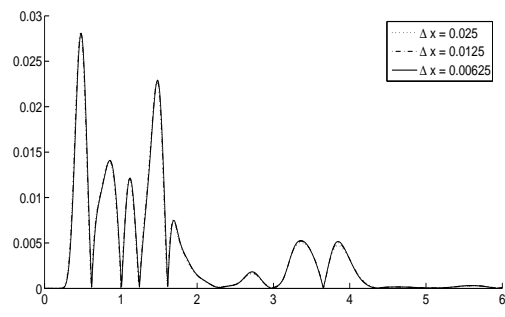

(b) $|v|((6.5,3.5) \mathrm{mm}, t), \beta=5 \varrho 10^{6}$

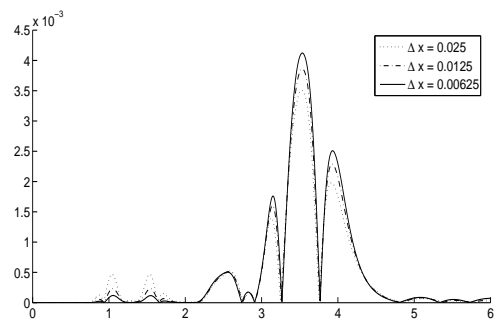

(d) $|v|((7.5,2.5) \mathrm{mm}, t), \beta=5 \varrho 10^{6}$

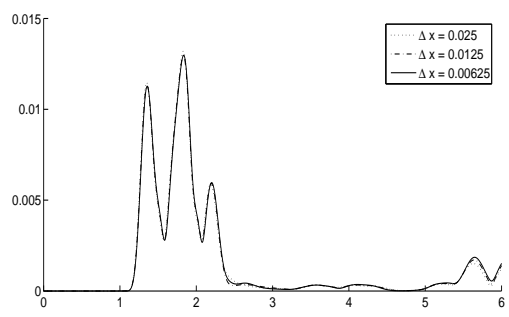

(f) $|v|((5.0,0.5), t), \beta=5 \varrho 10^{6}$

FIG. 4.4. The modulus of the velocity $|\mathbf{v}|\left(\mathbf{x}_{\mathbf{i}}, t\right), t \in[0,6], i \in\{1,2,3\}$, computed using the $Q_{1}^{\text {div }} \times P_{1}^{\text {disc }}$ element with $\beta=0$ on the left column and $\beta=5 \varrho 10^{6}$ on the right one.

error on those amplitudes is about $2 \%$ of the amplitude of the incident wave.

At the third point, the spurious phenomena are much stronger (see Figure 4.4, bottom left). Here the amplitude of the incident and scattered waves is about $40 \%$ of the amplitude of the actual incident wave. As we can observe, comparing the solutions obtained with the different meshes, the method converges very slowly (see the time interval $t \in[2,5.5] \mu \mathrm{s}$ ). This is due to the spurious modes created by the singularity on the tips of the crack (see also Figure 4.3(a)).

These spurious phenomena can be reduced using a positive value of $\beta$, the damping coefficient. We will explain how to chose $\beta$ in section 5.1. Let us analyze here the results obtained with $\beta=5 \varrho 10^{6}$. As we can see in Figure 4.3(b) the results seem to be better than those obtained with $\beta=0$ (see Figure 4.3(a)). The signal obtained at the first point $\mathbf{x}_{\mathbf{1}}$ is very similar for both choices (see Figure 4.4 , top right). The results for the second point are also comparable. We remark that some oscillations on the time intervals $t \in[2,3]$ and $t \in[4.5,6]$ are removed with the damping. It is in the signal recorded on the third point where the effect of the damping of the spurious modes is more efficient (see Figure 4.4, bottom right). The oscillations observed with 


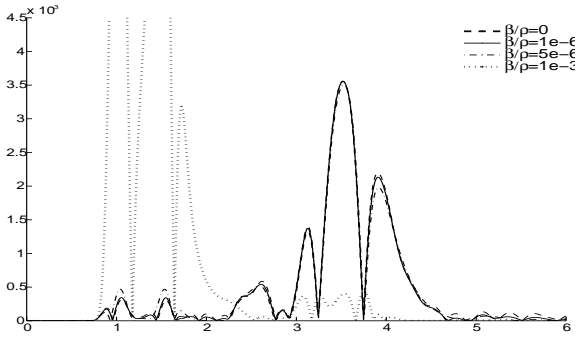

(a) $|v|((7.5,2.5) \mathrm{mm}, t)$

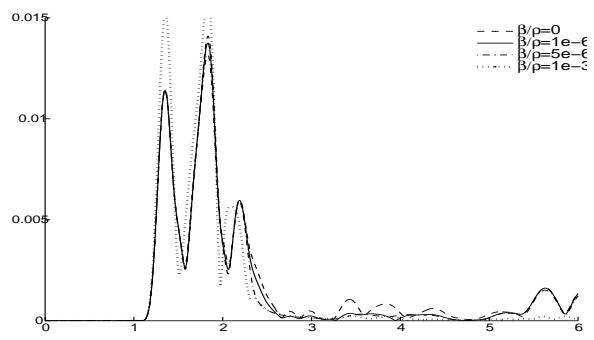

(b) $|v|((5.0,0.5) \mathrm{mm}, t)$

FIG. 4.5. The modulus of the velocity $|\mathbf{v}|\left(\mathbf{x}_{\mathbf{i}}, t\right), t \in[0,6] \mu \mathrm{s}, i \in\{2,3\}$, computed using the $Q_{1}^{\text {div }} \times P_{1}^{\text {disc }}$ element with $h=0.025 \mathrm{~mm}$ and different values of the damping parameter $\beta$.

$\beta=0$ are completely removed, and the method gives a good solution even with the coarsest mesh.

Influence of the damping parameter on the solution. To illustrate the effect of the damping parameter $\beta$ on the solution, we present in Figure 4.5 results obtained on the coarsest grid for different values of $\beta$. We have made the following observations: When we do not use any damping, the solution is polluted by spurious modes. On the other hand, the amplitude of the transmitted (nonphysical) waves through the crack increases as the value of the damping $\beta$ increases. This is expected, because the limit case $\beta \rightarrow+\infty$ corresponds to seeking the velocity in $Q_{0}$, and we know that in this case the method does not converge. There is thus an optimal value for the damping parameter $\beta$ to be determined so, as the spurious modes are damped, while the transmitted nonphysical wave remains small. In the next section we determine numerically the rate of convergence of the method for a particular geometry, and we discuss a procedure to choose the value of $\beta$.

5. Numerical error estimates. In this section we estimate numerically the order of convergence of the method. To do so, we consider solving the elastodynamic system on a disk $\Omega \subset \mathbb{R}^{2}$ with homogeneous Neumann boundary conditions on its boundary $\Gamma=\delta \Omega$. The geometry of the problem is presented in Figure 5.1.

To compute the solution we extend the unknowns in the domain of simple geometry $C$ (see Figure 5.1) and use the fictitious domain formulation (2.10) with zero
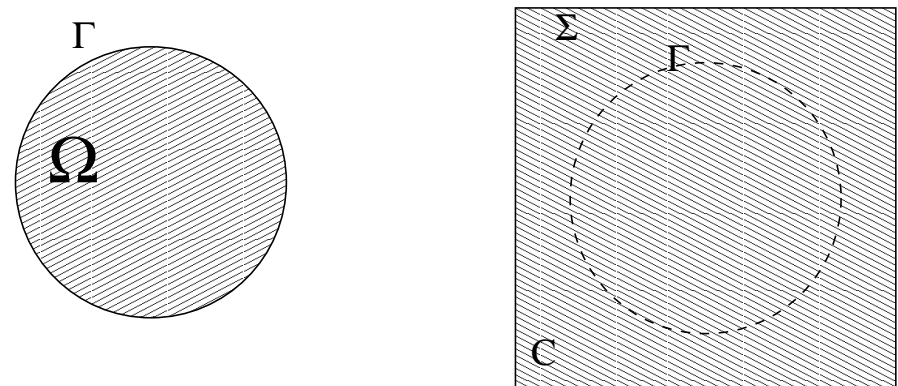

FIG. 5.1. The geometry of the problem. On the left is the initial domain of propagation $\Omega$, and on the right is the extended domain $C$ introduced by the fictitious domain formulation of the problem. 
force term $\mathbf{f}=0$ and an initial condition on the velocity field, given by

$$
\mathbf{v}((x, z), t=0)=0.1 F\left(\frac{r}{r_{0}}\right) \frac{\mathbf{r}+\mathbf{r}^{\perp}}{r}
$$

where $F(\cdot), \mathbf{r}$, and $r$ have been defined in section 3.2 and $r_{0}=1.5 \mathrm{~mm}$. The domain of propagation is an isotropic medium with the density and Lamé coefficients given by (3.8). The extended domain $C$ is truncated using perfectly matched layers $[11,16]$. We consider the final time equal to $T=5 \mu$ s when both the pressure and shear waves have reached the boundary.

The fact of having a problem that is rotationally invariant allows us to compute a reference solution solving a one-dimensional problem. More precisely, rewriting (2.1) in polar coordinates

$$
\mid \begin{array}{lll}
\rho \frac{\partial v_{r}}{\partial t}=\frac{\partial \sigma_{r r}}{\partial r}+\frac{1}{r} \frac{\partial \sigma_{r \theta}}{\partial \theta}+\frac{1}{r}\left(\sigma_{r r}-\sigma_{\theta \theta}\right) & & \text { in }[0, R] \times[0,2 \pi], \\
\rho \frac{\partial v_{\theta}}{\partial t}=\frac{\partial \sigma_{r \theta}}{\partial r}+\frac{1}{r} \frac{\partial \sigma_{\theta \theta}}{\partial \theta}+\frac{2}{r} \sigma_{r \theta} & & \text { in }[0, R] \times[0,2 \pi], \\
\frac{\partial \sigma_{r r}}{\partial t}=(2 \mu+\lambda) \frac{\partial v_{r}}{\partial r}+\lambda \frac{v_{r}}{r}+\lambda \frac{1}{r} \frac{\partial v_{\theta}}{\partial \theta} & & \text { in }[0, R] \times[0,2 \pi], \\
\frac{\partial \sigma_{\theta \theta}}{\partial t}=(2 \mu+\lambda)\left(\frac{1}{r} \frac{\partial v_{\theta}}{\partial \theta}+\frac{v_{r}}{r}\right)+\lambda \frac{\partial v_{r}}{\partial r} & & \text { in }[0, R] \times[0,2 \pi], \\
\frac{\partial \sigma_{r \theta}}{\partial t}=\mu\left(\frac{1}{r} \frac{\partial v_{r}}{\partial \theta}+\frac{\partial v_{\theta}}{\partial r}-\frac{v_{\theta}}{r}\right) & & \text { in }[0, R] \times[0,2 \pi], \\
\sigma_{r r}=0 \sigma_{r \theta}=0 & & \text { in } R \times[0,2 \pi],
\end{array}
$$

with the initial conditions

$$
v_{s}((r, \theta), t=0)=0.1 F\left(\frac{r}{r_{0}}\right), \quad s \in\{r, \theta\},
$$

we remark that the solution depends only on $r$, and the former equations are equivalent to the two following decoupled one-dimensional problems:

$$
\begin{array}{|l}
\mid \begin{array}{cc}
\rho \frac{\partial v_{r}}{\partial t}=\frac{\partial \sigma_{r r}}{\partial r}+\frac{1}{r}\left(\sigma_{r r}-\sigma_{\theta \theta}\right) & \text { in }[0, R], \\
\frac{\partial \sigma_{r r}}{\partial t}=(2 \mu+\lambda) \frac{\partial v_{r}}{\partial r}+\lambda \frac{v_{r}}{r} & \text { in }[0, R], \\
\frac{\partial \sigma_{\theta \theta}}{\partial t}=(2 \mu+\lambda) \frac{v_{r}}{r}+\lambda \frac{\partial v_{r}}{\partial r} & \text { in }[0, R], \\
\sigma_{r r}=0 & \text { in } R, \\
\frac{\partial v_{\theta}}{\partial t}=\frac{\partial \sigma_{r \theta}}{\partial r}+\frac{2}{r} \sigma_{r \theta} & \text { in }[0, R] \times[0,2 \pi], \\
\frac{\partial \sigma_{r \theta}}{\partial t}=\mu\left(\frac{\partial v_{\theta}}{\partial r}-\frac{v_{\theta}}{r}\right) & \text { in }[0, R] \times[0,2 \pi], \\
\sigma_{r \theta} y=0 & \text { in } R \times[0,2 \pi] .
\end{array}
\end{array}
$$

We solve numerically those systems using piecewise constant functions for the discretization of the velocity field and continuous linear functions for the stress tensor. 


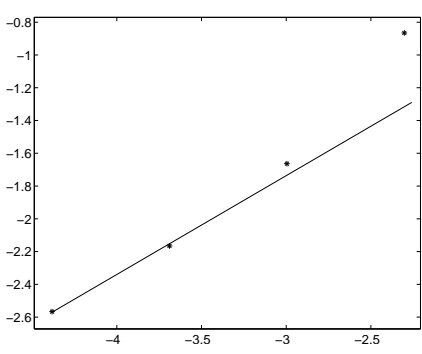

(a) $\sup _{t \leq T}\left\|\boldsymbol{\sigma}-\boldsymbol{\sigma}_{h}\right\|_{\underline{\underline{X}}}, s=0.59$

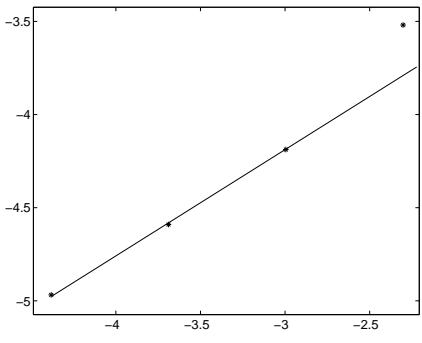

(b) $\sup _{t \leq T}\left\|\mathbf{v}-\mathbf{v}_{h}\right\|_{\underline{M}}, s=0.55$

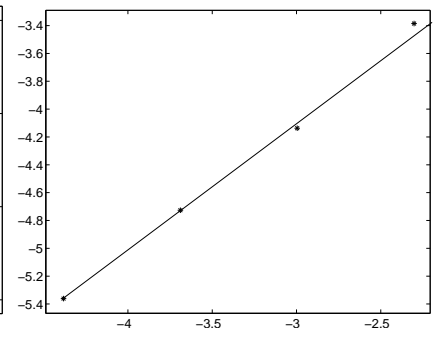

(c) $\sup _{t \leq T}\left\|\boldsymbol{\lambda}-\boldsymbol{\lambda}_{h}\right\|_{\underline{L^{2}}}, s=0.92$

FIG. 5.2. Numerical error on $\boldsymbol{\sigma}, \mathbf{v}$, and $\boldsymbol{\lambda}$ versus the discretization step.

For the time discretization we use a leapfrog scheme. The reference solution is obtained using a very fine mesh $\left(h_{1 d} \approx 1 / 800\right)$. The two-dimensional problem is solved using four different meshes with $h_{z}=h_{z}=1 / 10,1 / 20,1 / 40$, and $1 / 80$. We use the larger time step $\Delta t$ authorized by the CFL condition. The mesh for the object is uniform and with a discretization step $H$ such that $H / h \approx 4$ for each mesh. In all cases the damping parameter $\beta$ is equal to zero. For each numerical experiment we compute the difference between the approximated solution and the reference solution. In Figure 5.2 we display the logarithm of the error on the stress tensor, the velocity field, and the Lagrange multiplier versus the logarithm of the discretization step. The rate of convergence is thus given by the slope of the lines. We observe that the order of convergence for $\boldsymbol{\sigma}$ in $L^{\infty}\left([0, T],(H(\operatorname{div}, C))^{2}\right)$ norm and for $\mathbf{v}$ in $L^{\infty}\left([0, T],\left(L^{2}(C)\right)^{2}\right)$ norm are near the values we could expect (i.e., $1 / 2)$. The convergence rate obtained for the Lagrange multiplier $\boldsymbol{\lambda}$ (close to 1 ) is computed in $L^{\infty}\left([0, T] ;\left(L^{2}(\Gamma)\right)^{2}\right)$ norm, which means that we recover the conjectured convergence rate $(1 / 2)$ in $L^{\infty}\left([0, T] ;\left(H^{\frac{1}{2}}(\Gamma)\right)^{2}\right)$ norm.

In Figure 5.3 we display the same results but with the norm of the error now computed in $\widetilde{C}=C / B_{b}(\Gamma)$, i.e., the domain $C$ restricted from $B_{b}(\Gamma)$, defined by

$$
B_{b}(\Gamma)=\left\{\mathbf{x} \in C \text { subject to } \min _{\mathbf{y} \in \Gamma}|\mathbf{x}-\mathbf{y}| \leq b\right\}
$$

In this case, we observe that the convergence rate of the method is higher. This agrees with our intuition in the sense that the elements that we need to remove are the ones in which the solution has less regularity (see Remark 1), i.e., the elements that have nonzero intersection with the boundary $\Gamma$.

5.1. Determination of $\boldsymbol{\beta}$. Finally we discuss the influence of the damping parameter $\beta$ on the convergence results. In order to do so we repeat the experiment described above using the mesh with $h=1 / 40 \mathrm{~mm}$ for $\beta / \rho 10^{-6}=0,0.5,2.5,5,7.5,10$, 12.5, and 15. We display in Figure 5.4 the logarithm of the error on $\boldsymbol{\sigma}, \mathbf{v}$, and $\boldsymbol{\lambda}$ as a function of the value of $\beta / \rho 10^{-6}$. As we can see, better results are obtained for values of $\beta / \rho 10^{-6}$ between 2.5 and 5 . We also observe that the error increases when we choose $\beta$ too large. The same experiments done for different materials show that the optimal range for $\beta / \rho$ is independent of the material. It depends only on the number of points per wavelength in the discretization. It should be proportional to $1 / \Delta t$ :

$$
\frac{\beta}{\rho}=\frac{\zeta}{\Delta t}
$$




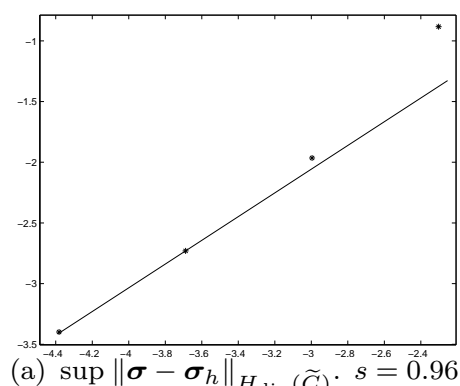

(a) $\sup _{t \leq T}^{-44}\left\|\boldsymbol{\sigma}^{-38}-\boldsymbol{\sigma}_{h}\right\|_{H_{\mathrm{div}}}^{-32}(\widetilde{C})^{-28} \cdot \stackrel{-26}{=} 0^{-24} .96$

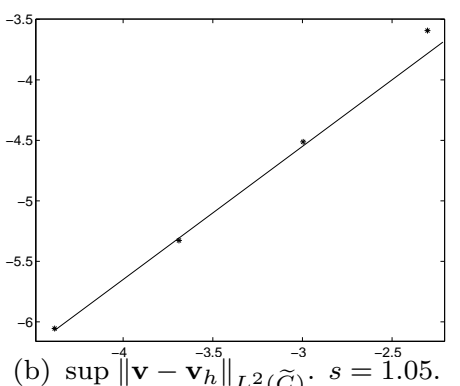

(b) $\sup _{t \leq T}\|\|^{-4} \mathbf{v}-\mathbf{v}_{h}^{-3.5} \|_{L^{2}}(\widetilde{C})^{-3} \cdot s=\stackrel{-2.5}{1} .05$.

Fig. 5.3. Numerical error $\boldsymbol{\sigma}, \mathbf{v}$ versus the discretization step. Here we compute the norm of the error in $\widetilde{C}$

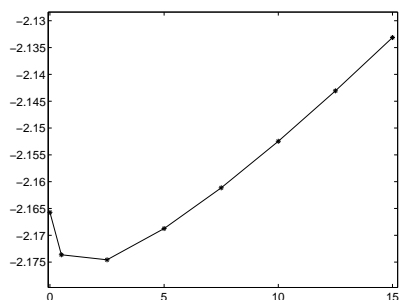

(a) $\sup _{t \leq T}\left\|\boldsymbol{\sigma}-\boldsymbol{\sigma}_{h}\right\|_{\underline{\underline{X}}}$.

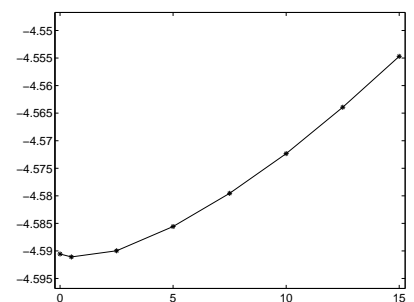

(b) $\sup _{t \leq T}\left\|\mathbf{v}-\mathbf{v}_{h}\right\|_{\underline{M}}$.

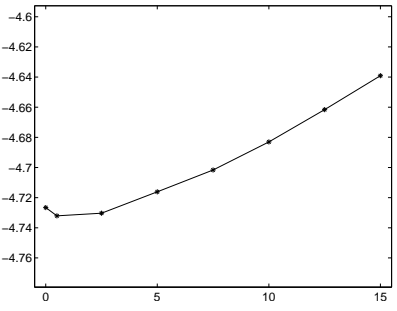

(c) $\sup _{t \leq T}\left\|\boldsymbol{\lambda}-\boldsymbol{\lambda}_{h}\right\|_{\underline{\mathcal{G}}}$.

FIG. 5.4. Numerical error on $\boldsymbol{\sigma}, \mathbf{v}$, and $\boldsymbol{\lambda}$ versus the damping parameter $\beta / \rho$.

where $\zeta$, an adimensional constant, determined by the previous experiment, should be chosen in the interval $[0.03,0.06]$.

Conclusions. In this paper we proposed a numerical method for solving the elastodynamic problem in anisotropic media with cracks (or more general objects) of complex geometry. Our approach leads to an efficient numerical method that uses regular grids for the space discretization (due to the fictitious domain formulation) and is explicit in time. The last point is achieved using a mixed finite element that is compatible with mass lumping for the discretization of the velocity-stress system. Although the theoretical convergence analysis is still an open problem, several numerical experiments suggest that the method provides a good approximate solution. 
Appendix. The matrix $\mathcal{K}_{h}$. We give here the expressions of the nonzero elements of the Hermitian matrix $\mathcal{K}_{h}$ that appears in the dispersion relation (4.5):

$$
\begin{aligned}
& \left(\mathcal{K}_{h}\right)_{1,1}=\frac{2}{\rho(k h)^{2}}\left(\mathcal{A} \sin ^{2}\left(\frac{k_{x} h}{2}\right)+\mathcal{B} \cos \left(k_{z} h\right) \sin ^{2}\left(\frac{k_{x} h}{2}\right)+2 \mathcal{D} \cos ^{2}\left(\frac{k_{x} h}{2}\right) \sin ^{2}\left(\frac{k_{z} h}{2}\right)\right), \\
& \left(\mathcal{K}_{h}\right)_{1,2}=\frac{-2 \mathbf{i} \mathcal{D}}{\sqrt{3} \rho(k h)^{2}} \sin \left(k_{x} h\right) \sin ^{2}\left(\frac{k_{z} h}{2}\right),\left(\mathcal{K}_{h}\right)_{1,3}=\frac{-2 \mathbf{i} \mathcal{B}}{\sqrt{3} \rho(k h)^{2}} \sin \left(k_{z} h\right) \sin ^{2}\left(\frac{k_{x} h}{2}\right), \\
& \left(\mathcal{K}_{h}\right)_{1,4}=\frac{\mathcal{C}+\mathcal{D}}{\rho(k h)^{2}} \sin \left(k_{x} h\right) \sin \left(k_{z} h\right),\left(\mathcal{K}_{h}\right)_{1,5}=\frac{-2 \mathbf{i} \mathcal{C}}{\sqrt{3} \rho(k h)^{2}} \sin \left(k_{z} h\right) \sin ^{2}\left(\frac{k_{x} h}{2}\right), \\
& \left(\mathcal{K}_{h}\right)_{1,6}=\frac{-2 \mathbf{i} \mathcal{D}}{\sqrt{3} \rho(k h)^{2}} \sin \left(k_{x} h\right) \sin ^{2}\left(\frac{k_{z} h}{2}\right),\left(\mathcal{K}_{h}\right)_{2,2}=\frac{4 \mathcal{D}}{3 \rho(k h)^{2}} \sin ^{2}\left(\frac{k_{x} h}{2}\right) \sin ^{2}\left(\frac{k_{z} h}{2}\right), \\
& \left(\mathcal{K}_{h}\right)_{2,4}=\frac{2 \mathbf{i} \mathcal{D}}{\sqrt{3} \rho(k h)^{2}} \sin \left(k_{z} h\right) \sin ^{2}\left(\frac{k_{x} h}{2}\right),\left(\mathcal{K}_{h}\right)_{2,6}=\frac{4 \mathcal{D}}{3 \rho(k h)^{2}} \sin ^{2}\left(\frac{k_{x} h}{2}\right) \sin ^{2}\left(\frac{k_{z} h}{2}\right), \\
& \left(\mathcal{K}_{h}\right)_{3,3}=\frac{2}{3 \rho(k h)^{2}}\left(\mathcal{A} \sin ^{2}\left(\frac{k_{x} h}{2}\right)-\mathcal{B} \cos \left(k_{z} h\right) \sin ^{2}\left(\frac{k_{x} h}{2}\right)\right), \\
& \left(\mathcal{K}_{h}\right)_{3,4}=\frac{2 \mathbf{i} \mathcal{C}}{\sqrt{3} \rho(k h)^{2}} \sin \left(k_{x} h\right) \sin ^{2}\left(\frac{k_{z} h}{2}\right),\left(\mathcal{K}_{h}\right)_{3,5}=\frac{-4 \mathcal{C}}{3 \rho(k h)^{2}} \sin ^{2}\left(\frac{k_{x} h}{2}\right) \sin ^{2}\left(\frac{k_{z} h}{2}\right), \\
& \left(\mathcal{K}_{h}\right)_{4,4}=\frac{2}{\rho(k h)^{2}}\left(\mathcal{A} \sin ^{2}\left(\frac{k_{z} h}{2}\right)+\mathcal{B} \cos \left(k_{x} h\right) \sin ^{2}\left(\frac{k_{z} h}{2}\right)+2 \mathcal{D} \cos ^{2}\left(\frac{k_{z} h}{2}\right) \sin ^{2}\left(\frac{k_{x} h}{2}\right)\right), \\
& \left(\mathcal{K}_{h}\right)_{4,5}=\frac{-2 \mathbf{i} \mathcal{B}}{\sqrt{3} \rho(k h)^{2}} \sin ^{2}\left(k_{x} h\right) \sin ^{2}\left(\frac{k_{z} h}{2}\right),\left(\mathcal{K}_{h}\right)_{4,6}=\frac{-2 \mathbf{i} \mathcal{D}}{\sqrt{3} \rho(k h)^{2}} \sin ^{2}\left(k_{z} h\right) \sin ^{2}\left(\frac{k_{x} h}{2}\right), \\
& \left(\mathcal{K}_{h}\right)_{5,5}=\frac{2}{3 \rho(k h)^{2}}\left(\mathcal{A} \sin ^{2}\left(\frac{k_{z} h}{2}\right)-\mathcal{B} \cos \left(k_{x} h\right) \sin ^{2}\left(\frac{k_{z} h}{2}\right)\right), \\
& \left(\mathcal{K}_{h}\right)_{6,6}=\frac{4 \mathcal{D}}{3 \rho(k h)^{2}} \sin ^{2}\left(\frac{k_{x} h}{2}\right) \sin ^{2}\left(\frac{k_{z} h}{2}\right),
\end{aligned}
$$

and the coefficients $\mathcal{A}, \mathcal{B}, \mathcal{C}$, and $\mathcal{D}$ are given by

$$
\mathcal{A}=\frac{8 \mu^{2}+8 \lambda \mu+\lambda^{2}}{2 \mu+\lambda}, \quad \mathcal{B}=\frac{\lambda^{2}}{2 \mu+\lambda}, \quad \mathcal{C}=\lambda, \quad \mathcal{D}=\mu .
$$

\section{REFERENCES}

[1] M. Amara And J.M. Thomas, Equilibrium finite elements for the linear elastic problem, Numer. Math., 33 (1979), pp. 367-383.

[2] D. N. Arnold, F. Brezzi, and J. Douglas, PEERS: A new mixed finite element for plane elasticity, Japan J. Appl. Math., 1 (1984), pp. 347-367.

[3] I. Babuska, The finite element method with Lagrangian multipliers, Numer. Math., 20 (1973), pp. 179-192.

[4] E. Bécache, A. Chaigne, G. Derveaux, and P. Joly, Time-domain simulation of a guitar: Model and method, J. Acoust. Soc. Amer., 6 (2003), pp. 3368-3383.

[5] E. BÉcAche, P. Joly, AND C. TsogkA, Eléments finis mixtes et condensation de masse en élastodynamique linéaire. (i) construction, C.R. Acad. Sci. Paris, Série I 325, (1997), pp. 545-550.

[6] E. BÉCAChe, P. Joly, And C. Tsogka, Etude d'un nouvel élément fini mixte permettant la condensation de masse, C.R. Acad. Sci. Paris, Série I 324, (1997), pp. 1281-1286.

[7] E. BÉcache, P. Joly, And C. Tsogka, An analysis of new mixed finite elements for the approximation of wave propagation problems, SIAM J. Numer. Anal., 37 (2000), pp. 10531084. 
[8] E. BÉCache, P. Joly, And C. Tsogka, Fictitious domains, mixed finite elements and perfectly matched layers for $2 d$ elastic wave propagation, J. Comput. Acoust., 9 (2001), pp. 11751203.

[9] E. BÉCache, P. Joly, and C. Tsogka, A new family of mixed finite elements for the linear elastodynamic problem, SIAM J. Numer. Anal., 39 (2002), pp. 2109-2132.

[10] E. BÉcache, J. Rodríguez, and C. Tsogka, On the convergence of the fictitious domain method for wave equation problems, Technical report 5802, INRIA, 2006.

[11] J. P. BÉREnger, A perfectly matched layer for the absorption of electromagnetic waves, J. Comput. Phys., 114 (1994), pp. 185-200.

[12] V.A. BoKIL AND R. Glowinski, An operator-splitting scheme with a distributed Lagrange multiplier based fictitious domain method for wave propagation problems, J. Comput. Phys., 205 (2005), pp. 242-268.

[13] F. Brezzi and M. Fortin, Mixed and Hybrid Finite Element Methods, Springer-Verlag, New York, 1991.

[14] G. Cohen, Higher-order numerical methods for transient wave equations, Sci. Comput. XIII, Springer-Verlag, Berlin, 2002, with a foreword by R. Glowinski.

[15] F. Collino, P. Joly, And F. Millot, Fictitious domain method for unsteady problems: Application to electromagnetic scattering, J. Comput. Phys., 138 (1997), pp. 907-938.

[16] F. Collino And C. Tsogka, Application of the PML absorbing layer model to the linear elastodynamic problem in anisotropic heteregeneous media, Geophysics, 66 (2001), pp. 294307.

[17] S. Garcès, Application des Méthodes de Domaines Fictifs à la Modélisation des Structures Rayonnantes Tridimensionnelles, Ecole Nationale Supérierure de l'Aéronautique et de l'Espace, 1997.

[18] V. Girault And R. Glowinski, Error analysis of a fictitious domain method applied to a Dirichlet problem, Japan J. Indust. Appl. Math., 12 (1995), pp. 487-514.

[19] V. GiRault And P. A. RAVIART, Finite element methods for Navier-Stokes equations, Springer Ser. Comput. Math. 5, Springer-Verlag, Berlin, 1986, theory and algorithms.

[20] R. Glowinski And Y. Kuznetsov, On the solution of the Dirichlet problem for linear elliptic operators by a distributed Lagrange multiplier method, C. R. Acad. Sci. Paris Sér. I Math., 327 (1998), pp. 693-698.

[21] R. Glowinski, T. W. Pan, T. I. Hesla, D. D. Joseph, and J. Periaux, A distributed Lagrange multiplier/fictitious domain method for flows around moving rigid bodies: Application to particulate flow, Internat. J. Numer. Methods Fluids, 30 (1999), pp. 1043-1066.

[22] R. Glowinski, T. W. Pan, T. I. Hesla, D. D. Joseph, and J. Periaux, A distributed Lagrange multiplier/fictitious domain method for the simulation of flow around moving rigid bodies: Application to particulate flow, Comput. Methods Appl. Mech. Engrg., 184 (2000), pp. 241267.

[23] R. Glowinski, T. W. Pan, T. I. Hesla, D. D. Joseph, and J. Periaux, a fictitious domain approach to the direct numerical simulation of incompressible viscous fluid past moving rigid bodies: Application to particulate flow, J. Comput. Methods Phys., 169 (2001), pp. 363-426.

[24] R. Glowinski, T. W. Pan, and J. Periaux, A fictitious domain method for Dirichlet problem and applications, Comput. Methods Appl. Mech. Engrg., 111 (1994), pp. 283-303.

[25] R. Glowinski, T. W. Pan, and J. Periaux, A fictitious domain method for external incompressible flow modeled by navier-stokes equations, Comput. Methods Appl. Mech. Engrg., 112 (1994), pp. 133-148.

[26] P. Grisvard, Problèmes aux limites dans les polygones. Mode d'emploi, EDF Bull. Direction Études Rech. Sér. C. Math. Inform., 1 (1986), pp. 21-59.

[27] E. Heikkola, Y. A. Kuznetsov, P. Neittaanmäki, and J. Toivanen, Fictitious domain methods for the numerical solution of two-dimensional scattering problems, J. Comput. Phys., 145 (1998), pp. 89-109.

[28] C. Johnson And B. Mercier, Some equilibrium finite element methods for two-dimensional elasticity problems, Numer. Math., 30 (1978), pp. 103-116.

[29] P. Joly and L. Rhaouti, Analyse numérique - Domaines fictifs, éléments finis H(div) et condition de Neumann : le problème de la condition inf-sup, C.R. Acad. Sci. Paris, Série I 328, (1999), pp. 1225-1230.

[30] Y. A. KuZnetsov, Fictitious component and domain decomposition methods for the solution of eigenvalue problems, in Proceedings of the Seventh International Symposium on Computing Methods in Applied Sciences and Engineering Versailles, 1985, North-Holland, Amsterdam, 1986 , pp. $155-172$.

[31] M. E. Morley, A family of mixed finite elements for linear elasticity, Numer. Math., 55 (1989), 
pp. $633-666$.

[32] J.C. NÉDÉLEC, A new family of mixed finite elements in $\mathbb{R}^{3}$, Numer. Math., 50 (1986), pp. 57 81.

[33] L. Rhaouti, Domaines Fictifs pour la Modélisation d'un Probème d' Interaction FluideStructure: Simulation de la Timbale, Ph.D. thesis, Université Paris IX, 1999.

[34] R. Stenberg, On the construction of optimal mixed finite element methods for the linear elasticity Problem, Numer. Math., 48 (1986), pp. 447-462.

[35] R. Stenberg, A family of mixed finite elements for the elasticity problem, Numer. Math., 53 (1988), pp. 513-538.

[36] N. Torduman, Eléments Finis d'Ordre Élevé vec Condensation de Masse pour l'Équation des Ondes, Ph.D. thesis, Université Paris IX, 1995. 